\title{
Supporting Information For: Conformational Analysis of Chiral Helical Perfluoroalkyl Chains by VCD
}

\author{
Kenji Monde, ${ }^{*, \dagger}$ Nobuaki Miura, ${ }^{\dagger}$ Mai Hashimoto, ${ }^{\dagger}$ Tohru Taniguchi, $^{\dagger}$ \\ Tamotsu Inabe \\ ${ }^{\dagger}$ Division of Biological Sciences, Graduate School of Science, \\ Frontier Research Center for Post-Genomic Science and Technology, \\ Hokkaido University, Kita 21 Nishi11, \\ Sapporo 001-0021, Japan \\ ${ }^{7}$ Division of Chemistry, Graduate School of Science, \\ Hokkaido University, Kita10, Nishi 8, \\ Sapporo 060-0810, Japan
}

\section{General Procedures.}

${ }^{1} \mathrm{H}$ NMR (600 or $500 \mathrm{MHz}$ ) and ${ }^{13} \mathrm{C}$ NMR (125 MHz) spectra were recorded on a Bruker Avance spectrometer with $\mathrm{CDCl}_{3}$ or DMSO- $d_{6}$ as solvents. Chemical shifts $(\delta)$ are reported in ppm downfield from TMS, and the coupling constants $(J)$ are given in Hz. The FAB-MS and HR-MS were measured on a JEOL JMS-HX110. Analytical TLC was performed on $0.2 \mathrm{~mm}$ silica gel plates (Merck 60 F-254) and column chromatography was carried out with silica gel (Kanto $60 \mathrm{~N}, 40-50 \mu \mathrm{m}$ ). A Shimadzu LC-6A Liquid Chromatograph instrument equipped with a Shimadzu SPD-6AV UV-VIS Spectrometric Detector was used to perform optical resolutions. A Hitachi D-2520 GPC-Integrator was used to depict elution curves. Optical rotations were measured at room temperature in a $1 \mathrm{~cm}$ cell with a PERKIN ELMER Polarimeter 343 at the sodium D-line. UV and CD spectra were obtained in a $1 \mathrm{~mm}$ quartz cell on a JASCO J-810 spectrometer. The molar extinction coefficient $\varepsilon$ of IR and UV spectra are given in $\mathrm{cm}^{2} \mathrm{mmol}$. IR and VCD spectra of each enantiomer were measured using Bomem/BioTools Chiralir and JASCO JV-2001 spectrometers. The samples were dissolved in $\mathrm{CDCl}_{3}$ or DMSO- $d_{6}$ (concentration: $0.04 \mathrm{M}$ for 1 and $0.06 \mathrm{M}$ for 2) and then placed in a $\mathrm{BaF}_{2}$ or $\mathrm{CaF}_{2}$ cell (pathlength: $71 \mu \mathrm{m}$ for $\mathbf{1}$ and $100 \mu \mathrm{m}$ for 2), and the VCD spectra were recorded for $3 \mathrm{~h}$ at an $8 \mathrm{~cm}^{-1}$ resolution. Spectroscopic solvent grades were purchased from Wako Pure Chemical Industries, Ltd., and $\mathrm{CDCl}_{3}$, DMSO- $d_{6}$ was obtained from Cambridge Isotope Laboratories. $1 H, 1 H, 9 H$-hexadecafluoro-1-nonanol and perfluorooctyl iodide were purchased from Daikin Industries, Ltd. The single-crystal X-ray diffraction measurements via an automated Rigaku RAXIS RAPID

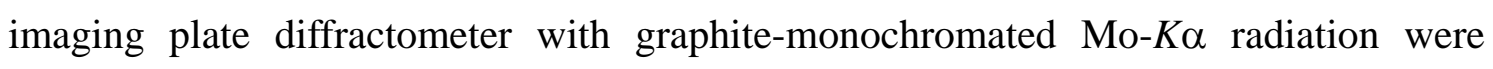
performed for the crystals of $(R)-(-)-2$. The measurements were carried out at $123 \mathrm{~K}$ 
with cold nitrogen gas flow equipment. The structure was solved for by a direct method, and the hydrogen atoms were placed at the calculated ideal positions.

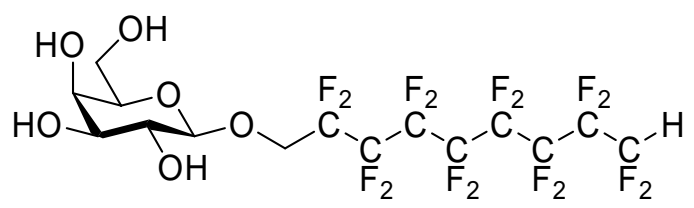

\section{Synthesis of (9H-hexadecafluorooctyl)methyl $\beta$-D-galactopyranoside (1)}

To a solution of peracetyl $\beta$-D-galactopyranose $(1.00 \mathrm{~g}, 2.56 \mathrm{mmol})$ in dichloromethane $(10 \mathrm{ml})$ was added $1 H, 1 H, 9 H$-hexadecafluoro-1-nonanol (2.21 g, 5.12 mmol) and boron trifluoride diethyl ether complex $(650 \mu \mathrm{l}, 5.12 \mathrm{mmol})$ at $0{ }^{\circ} \mathrm{C}$. The mixture was stirred at $0{ }^{\circ} \mathrm{C}$ for $2 \mathrm{~h}$, and kept at room temperature overnight. After addition of dichloromethane, the mixture was washed with saturated $\mathrm{NaHCO}_{3}$ and brine. The organic solution was dried over $\mathrm{MgSO}_{4}$, filtered, and then the filtrate was evaporated in vacuo. The residue was chromatographed on silica gel (toluene-ethyl acetate, 5:1) to afford a tetraacetate of $\mathbf{1}$, ( $9 H$-hexadecafluorooctyl)methyl 2,3,4,6-tetra-O-acetyl- $\beta$-D-Galactopranoside) as a white solid (1.25 g, 64\%).

Tetraacetate of 1: mp $68-70^{\circ} \mathrm{C}$ (hexane); $[\alpha]_{\mathrm{D}}{ }^{25}-6.1$ (c 1.0, $\left.\mathrm{CHCl}_{3}\right)$; IR $\left(\mathrm{CHCl}_{3}\right) 1751$, 1371, 1223, 1146, 1082, $1063 \mathrm{~cm}^{-1} ;{ }^{1} \mathrm{H}$ NMR (600 MHz, $\left.\mathrm{CDCl}_{3}\right) \delta 6.06$ (tt, $J=52.2$, $4.8 \mathrm{~Hz}, 1 \mathrm{H} ; \mathrm{CF}_{2} \mathrm{H}$ ), 5.41 (dd, $J=3.3,1.2 \mathrm{~Hz}, 1 \mathrm{H} ; 4-\mathrm{H}$ ), 5.25 (dd, $J=10.2,7.8 \mathrm{~Hz}, 1 \mathrm{H}$; 2-H), 5.03 (dd, $J=3.6,10.2 \mathrm{~Hz}, 1 \mathrm{H} ; 3-\mathrm{H}), 4.61$ (d, $J=8.4 \mathrm{~Hz}, 1 \mathrm{H} ; 1-\mathrm{H}), 4.32$ (dt, $J=12.6,12.6 \mathrm{~Hz}, 1 \mathrm{H} ; \mathrm{CH}_{2}$ ), 4.20-4.13 (m, 2H; 6-Ha,b), 4.06 (dt, $J=12.6,12.6 \mathrm{~Hz}, 1 \mathrm{H}$; $\mathrm{CH}_{2}$ ), 3.94 (dt, $J=6.6,0.6 \mathrm{~Hz}, 1 \mathrm{H}$; 5-H), 2.16 (s, 3H; Ac), 2.06 (s, 3H; Ac), 2.04 (s, 3H; Ac), 1.99 (s, 3H; Ac); ${ }^{13} \mathrm{C}$ NMR (125 MHz, $\mathrm{CDCl}_{3}$ ): $\delta$ 170.32, 170.12, 170.05, 169.27, 101.55, 71.12, 70.52, 68.18, 66.78, 65.27 (t), 61.12, 20.58, 20.51, 20.31; MS (FAB): $\mathrm{m} / \mathrm{z}$ (\%): 855 (10) $\left[\mathrm{M}+\mathrm{C}_{3} \mathrm{H}_{8} \mathrm{O}_{3}+\mathrm{H}\right]^{+}, 761(10)[\mathrm{M}-\mathrm{H}]^{+}, 331(100)\left[\mathrm{M}-\left(\mathrm{H}\left(\mathrm{CF}_{2}\right)_{8} \mathrm{CH}_{2} \mathrm{O}\right)\right]^{+}, 289$ (10) $\left[\mathrm{M}-\left(\mathrm{H}\left(\mathrm{CF}_{2}\right)_{8} \mathrm{CH}_{2} \mathrm{O}\right)-\mathrm{CH}_{3} \mathrm{CO}+\mathrm{H}\right]^{+}, 93$ (20) $\left[\mathrm{C}_{3} \mathrm{H}_{9} \mathrm{O}_{3}\right]^{+}$; HRMS (FAB): calcd for $\mathrm{C}_{23} \mathrm{H}_{21} \mathrm{~F}_{16} \mathrm{O}_{10}$ 761.0879, found 761.0895.

To a solution of tetraacetate of 1 (100 $\mathrm{mg}, 0.131 \mathrm{mmol})$ in methanol $(5 \mathrm{ml})$ was added sodium methoxide ( $2 \mathrm{mg}, 0.03 \mathrm{mmol}$, catalytic amounts). The mixture was stirred at room temperature for $1 \mathrm{~h}$. The reaction mixture was then neutralized with Dowex 50W-X8 $\left[\mathrm{H}^{+}\right]$resin. The reaction mixture was filtered, and then the filtrate was evaporated in vacuo to give almost pure 1 as a white solid (77 mg, quant).

1: mp $144-154^{\circ} \mathrm{C}$ (methanol); $[\alpha]_{\mathrm{D}}{ }^{25}-13.8$ (c 1.0, DMSO); IR (DMSO- $d_{6}$ ) 3337, 1212, 1183, 1165, 1150, $1107 \mathrm{~cm}^{-1}$; ${ }^{1} \mathrm{H}$ NMR (600 MHz, DMSO-d $\left.{ }_{6}\right) \delta 7.22$ (tt, $J=60.1,6.0$ 
$\mathrm{Hz}, 1 \mathrm{H} ; \mathrm{CF}_{2} \mathrm{H}$ ), 5.15 (d, $\left.J=6.0 \mathrm{~Hz}, 1 \mathrm{H} ; 2-\mathrm{OH}\right), 5.03$ (d, $\left.J=6.0 \mathrm{~Hz}, 1 \mathrm{H} ; 3-\mathrm{OH}\right), 4.97$ (d, $J=6.0 \mathrm{~Hz}, 1 \mathrm{H} ; 4-\mathrm{OH}), 4.54$ (t, $J=7.1 \mathrm{~Hz}, 1 \mathrm{H} ; 6-\mathrm{OH}), 4.41$ (dt, $J=17.6,16.5 \mathrm{~Hz}, 1 \mathrm{H}$; $\mathrm{CH}_{2}$ ), 4.32 (d, $J=9.3 \mathrm{~Hz}, 1 \mathrm{H} ; 1-\mathrm{H}$ ), 4.25 (dt, $J=17.2,16.5 \mathrm{~Hz}, 1 \mathrm{H} ; \mathrm{CH}_{2}$ ), 3.68 (br dd, $J$ =13.1, $8.3 \mathrm{~Hz}, 1 \mathrm{H} ; 6-\mathrm{Hb}), 3.47-3.38$ (m, 1H; 6-Ha), 3.16-3.13 (m, 2H; 3-H, 5-H), 2.99-3.07 (m, 2H; 2-H, 4-H); ${ }^{13} \mathrm{C}$ NMR (125 MHz, DMSO-d $\left.{ }_{6}\right) \delta 102.56,77.15,76.59$, 73.08, 69.85, 63.84 (t), 61.03; MS (FAB): m/z (\%): 617 (40) [M+Na] $]^{+}, 325$ (30), 255

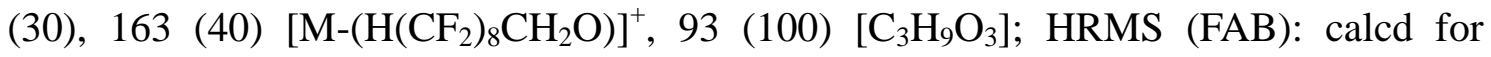
$\mathrm{C}_{15} \mathrm{H}_{14} \mathrm{~F}_{16} \mathrm{O}_{6} \mathrm{Na}$ 617.0433; found 617.0425.<smiles>OC(c1ccccc1)C(F)CCC(F)CC(F)CC(F)F</smiles>

\section{Synthesis of racemic perfluorooctylphenylmethanol (2)}

Under a $\mathrm{N}_{2}$ atmosphere, a 1.6 M solution of methyllithium-lithium bromide in diethyl ether $(2.75 \mathrm{ml}, 4.4 \mathrm{mmol})$ was added to a solution of benzaldehyde (314 $\mathrm{mg}, 2.95$ $\mathrm{mmol}$ ) and perfluorooctyl iodide $(2.96 \mathrm{~g}, 5.41 \mathrm{mmol})$ in diethyl ether $(6 \mathrm{ml})$ at $-78^{\circ} \mathrm{C}$. The mixture was stirred at the same temperature for $15 \mathrm{~min}$, and kept at $-40^{\circ} \mathrm{C}$ overnight. Small amount of methanol and water $(5 \mathrm{ml})$ were added to the reaction solution, and extracted with diethyl ether. The combined extract was washed with water. The extract was dried over $\mathrm{MgSO}_{4}$, filtered, and then the filtrate was evaporated in vacuo. The residue was purified by column chromatography on silica gel (hexane-ethyl acetate, 20:1) to give racemic $\mathbf{2}^{1}$ as a white solid (514 $\mathrm{mg}$, 33\%).

2: ${ }^{1} \mathrm{H}$ NMR (600 MHz, $\left.\mathrm{CDCl}_{3}\right) \delta 7.48$ (m, 2H; Ph), 7.43 (m, 3H; Ph), 5.22 (ddd, $J=$ 17.7, 5.7, $5.7 \mathrm{~Hz}, 1 \mathrm{H} ; \mathrm{CH}), 2.45$ (d, $J=5.2 \mathrm{~Hz}, 1 \mathrm{H}$; OH).

(1) Linderman, R. J.; Graves, D. M.; J. Org. Chem. 1989, 54, 661.

\section{Optical resolution of 2 by the HPLC on the chiral column.}

A preparative HPLC was performed on a CHIRALCEL ${ }^{\circledR}$ OD column $(1 \mathrm{~cm} \phi$ x 25 $\mathrm{cm})$ with hexane : ethanol $=6: 1$ at a flow rate of $4 \mathrm{~mL} / \mathrm{min}\left(\mathrm{t}_{0}=3.77, \mathrm{t}_{1}=5.24, \mathrm{t}_{2}=\right.$ $6.25 \mathrm{~min} ; \alpha=1.69)$. The first eluted $(R)-(-)-2:[\alpha]_{\mathrm{D}}{ }^{25}-13.2\left(c 1.0, \mathrm{CHCl}_{3}\right)$, the second eluted $(S)-(+)-2:[\alpha]_{\mathrm{D}}{ }^{25}+13.3\left(\right.$ ( $\left.1.0, \mathrm{CHCl}_{3}\right)$. 


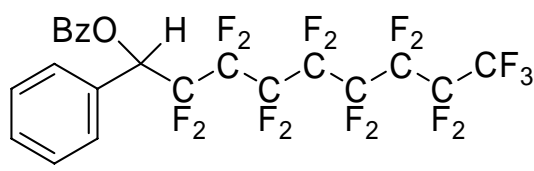

\section{Synthesis of perfluorooctylphenylmethyl benzoate (3)}

To a solution of perfluorooctylphenyl methanol (-)-2 (20 mg, $38 \mu \mathrm{mol})$ in dry pyridine $(200 \mu \mathrm{l})$ was added benzoyl chloride (80 $\mu \mathrm{l}, 690 \mu \mathrm{mol})$. The mixture was stirred overnight at room temperature. The mixture was extracted with chloroform. The organic layer was washed with $1 \mathrm{~N} \mathrm{HCl}$, saturated $\mathrm{NaHCO}_{3}$, brine and dried over $\mathrm{MgSO}_{4}$. The solvent was removed in vacuo and purifed by PTLC (hexane-ethyl acetate, 5:1) to give (+)-3 (14 mg, 58\%) as a white solid. An antipode (+)-2 was converted to a corresponding benzoate (-)-3 by the same procedure. Absolute configurations of $(+)-3$ and (-)-3 were determined by comparison to the previously reported method. ${ }^{2}$ (R)-(+)-3: mp 82-84 ${ }^{\circ} \mathrm{C}\left(\mathrm{CHCl}_{3}\right) ;[\alpha]_{\mathrm{D}}^{25}+38.6$ (c 1.0, $\left.\mathrm{CHCl}_{3}\right)$; IR $\left(\mathrm{CHCl}_{3}\right)$ 1738, 1712, 1452, 1360, 1242, 1219, 1207, 1151, 1095, 1068, $1030 \mathrm{~cm}^{-1}$; UV-Vis $\left(\mathrm{CH}_{3} \mathrm{OH}\right): \lambda_{\max }$ $231 \mathrm{~nm}, \varepsilon 14200 \mathrm{M}^{-1} \mathrm{~cm}^{-1}$; CD $\left(\mathrm{CH}_{3} \mathrm{OH}\right): \lambda_{\text {ext }} 230 \mathrm{~nm}, \Delta \varepsilon+8.8 \mathrm{M}^{-1} \mathrm{~cm}^{-1}$; ${ }^{1} \mathrm{H}$ NMR $(600$ $\left.\mathrm{MHz} \mathrm{CDCl}_{3}\right) \delta 8.11$ (d, $\left.J=7.2 \mathrm{~Hz}, 2 \mathrm{H} ; \mathrm{Ph}\right), 7.63$ (t, $\left.J=7.5 \mathrm{~Hz}, 1 \mathrm{H} ; \mathrm{Ph}\right), 7.55-7.52$ (m, 2H; Ph), 7.49 (t, $J=7.8$ Hz, 2H; Ph), 7.43-7.40 (m, 3H; Ph), 6.51 (dd, $J=18.4,5.7$ Hz, $1 \mathrm{H} ; \mathrm{CH}) ;{ }^{13} \mathrm{C}$ NMR (125 MHz, $\left.\mathrm{CDCl}_{3}\right): \delta$ 163.98, 133.90, 130.89, 130.01 128.67, 128.57, 128.54 ; MS (FAB): m/z (\%): 631 (10) [M+H] ${ }^{+}, 509$ (90) [M-C $\left.\mathrm{H}_{6} \mathrm{H}_{5} \mathrm{COO}\right]^{+}, 154$ (35) $\left[\mathrm{C}_{7} \mathrm{H}_{7} \mathrm{NO}_{3}+\mathrm{H}\right]^{+}, 137$ (40) $\left[\mathrm{C}_{7} \mathrm{H}_{7} \mathrm{NO}_{3}-\mathrm{OH}\right]^{+}, 105$ (65); HRMS (FAB): calcd for $\mathrm{C}_{22} \mathrm{H}_{12} \mathrm{~F}_{17} \mathrm{O}_{2}$ 631.0566, found 631.0560.

(S)-(-)-3: mp 85-86 ${ }^{\circ} \mathrm{C}\left(\mathrm{CHCl}_{3}\right)$; $[\alpha]_{\mathrm{D}}{ }^{25}-38.0$ (c 1.0, $\left.\mathrm{CHCl}_{3}\right)$; UV-Vis $\left(\mathrm{CH}_{3} \mathrm{OH}\right) \lambda_{\max }$ $231 \mathrm{~nm}, \varepsilon 14000 \mathrm{M}^{-1} \mathrm{~cm}^{-1}$; $\mathrm{CD}\left(\mathrm{CH}_{3} \mathrm{OH}\right) \lambda_{\text {ext }} 230 \mathrm{~nm}, \Delta \varepsilon-7.9 \mathrm{M}^{-1} \mathrm{~cm}^{-1}$.

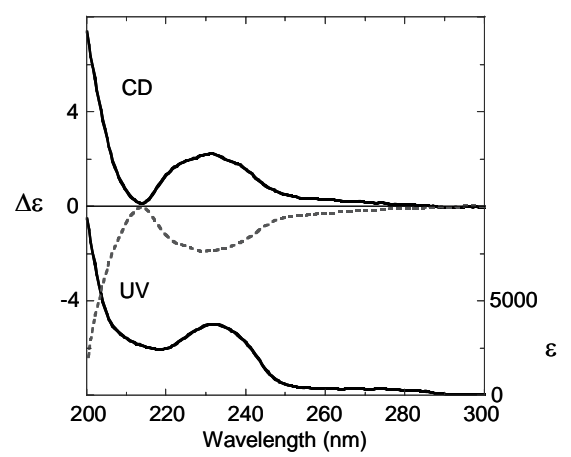

Figure S1. UV and CD spectra of benzoates $(R)-(+)-3$ (solid line) and (S)-(-)-3 (dotted line) in $\mathrm{CH}_{3} \mathrm{OH}$ derived from the first eluted $(R)-(-)-2$ and the second eluted (S)-(+)-2, respectively.

(2) Adam, W.; Lukacs, Z.; Viebach, K.; Humpf, H. -U.; Saha-Möller, C. R.; Schreier, P. J. Org. Chem. 2000, 65, 186. 


\section{$X$-ray crystallographic study of (R)-(-)-2.}

Careful recrystallization of $(R)-(-)-2$ from hexane afforded colorless prisms suitable for the X-ray analysis. Crystal data for $(R)-(-)-2$ : colorless needle, $0.45 \times 0.06 \times 0.05$ $\mathrm{mm}^{3}, \mathrm{C}_{15} \mathrm{H}_{7} \mathrm{~F}_{17} \mathrm{O}_{1}, M=526.19$, monoclinic, space group $P 2_{1}, a=10.586(8), b=$ 5.241(3), $c=16.901(12) \AA, \beta=105.81(3)^{\circ}, V=902.1(10) \AA^{3}, T=123(1) \mathrm{K}, Z=2, \rho_{\text {calc }}$ $=1.937 \mathrm{~g} \mathrm{~cm}^{-3}, \mu=0.240 \mathrm{~mm}^{-1}, 2 \theta_{\max }=55^{\circ}, \mathrm{Mo}_{\mathrm{K} \alpha}(\lambda=0.71075 \AA)$, Rigaku RAXIS RAPID imaging plate diffractometer. A total of 7704 reflections were measured, of which 3751 reflections were unique $\left(R_{\mathrm{int}}=0.055\right)$. Anisotropic thermal parameters for non-hydrogen atoms and isotropic ones for hydrogen. Final $R 1=0.069\left(F^{2}>2 \sigma\left(F^{2}\right)\right.$ and $w R 2=0.138$ (on $F^{2}$; all data; 306 parameters) with GOF $=1.018$, maximum residual peak 0.96 e $\AA^{-3}$, minimum residual peak -0.68 e $\AA^{-3}$. CCDC-289473 contains the supplementary crystallographic data for this paper. These data can be obtained free of charge from the Cambridge Crystallographic Data Centre via www.ccdc.cam.ac.uk/data_request/cif.

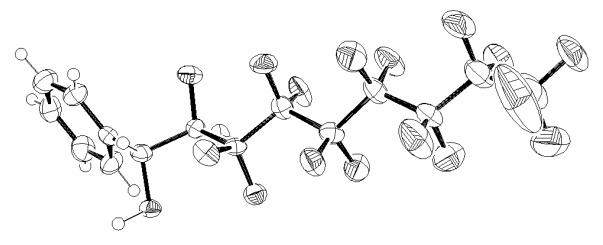

Figure S2. (a) An ORTEP drawing of (R)-(-)-2 with 50\% probability ellipsoids. 


\section{Computation.}

\section{Theoretical calculation of $C_{8} F_{18}(4)$.}

Geometrical optimizations and a harmonic frequency analysis were performed using DFT calculations at the B3PW91/6-31G(d,p) level of theory. Using the obtained frequencies and intensities of the IR and VCD spectra, the convoluted spectra with Lorenz functions whose half widths at half maximum were $10 \mathrm{~cm}^{-1}$ were calculated for the two lowest conformers.

(4)-MI

$$
M \text { (left-handed) }
$$

(4) $-M 2$

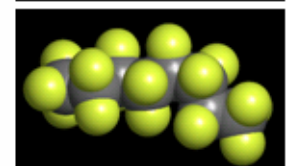

(4) $-M 3$

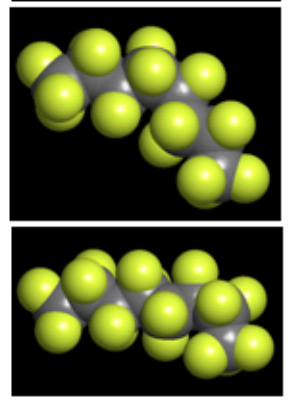

(4)-M4
$0.00(0.00)$

$30.2 \%(33.6 \%)$

$0.58(0.71)$

$11.4 \%(10.1 \%)$

$1.31(1.40)$

$3.3 \%(3.1 \%)$

$0.66(0.95)$

$9.9 \%(6.7 \%)$ relative energy (by free energy) Ratio (by free energy)

(4) $-P I$

$P$ (right-handed)

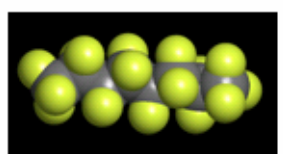

$0.00(0.00)$

$30.2 \%(33.2 \%)$

(4)-P2

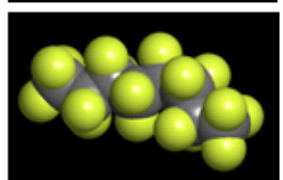

$1.42(1.24)$

$2.7 \%(4.1 \%)$

(4) $-P 3$

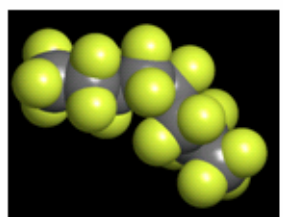

$0.64(0.92)$

$10.1 \%(7.1 \%)$

(4) $-P 4$

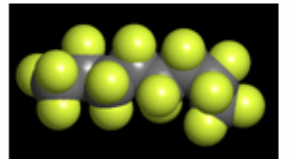

$1.59(1.68)$

$2.1 \%(2.0 \%)$

Figure S3. Low-lying conformers of $P$ (right-handed)- and $M$ (left-handed)- $\mathrm{C}_{8} \mathrm{H}_{18}$ (4). Upper numbers indicate relative energies in $\mathrm{kcal} / \mathrm{mol}$ and free energies in $\mathrm{kcal} / \mathrm{mol}$ in parentheses, while lower numbers indicate their corresponding ratios and ones by their free energies in parentheses.

\section{Theoretical calculation of (R)-pefluorohexylphenylmethanol (2).}

In the conformational search, the C-C-C-C dihedral angle in the perfluoro alkyl chain was fixed at $180^{\circ}$. In addition, the $6-31+G(d, p)$ basis sets were used due to its slightly complex structure. The IR and VCD spectra of each conformation were calculated by convolution with Lorenz functions whose half widths at half maximum were $10 \mathrm{~cm}^{-1}$ using the frequency and the intensity of the spectra. Averaged IR and VCD spectra were calculated with the Boltzmann weighted populations for the lower twelve conformers. 
Table S1. Total energy, relative energy, and Boltzmann weighted population for each conformer of $(R)-2$. $P$ and $M$ indicates its helicity of each conformer ( $P$ : right-handed conformer, $M$ : left-handed conformer).

\begin{tabular}{|c|c|c|c|}
\hline conformation & Total Energy & $\Delta E$ & Boltzmann \\
\hline & (au) & (kcal/mol) & weight \\
\hline (2)-P 3 & -2347.366727 & 0.00 & 0.251 \\
\hline (2)-P 6 & -2347.366467 & 0.16 & 0.190 \\
\hline (2)-M 1 & -2347.366300 & 0.27 & 0.160 \\
\hline (2)-M 6 & -2347.365997 & 0.46 & 0.116 \\
\hline (2)-M 3 & -2347.365513 & 0.76 & 0.069 \\
\hline (2)-P 4 & -2347.365349 & 0.86 & 0.058 \\
\hline (2)- $P 1$ & -2347.365221 & 0.94 & 0.051 \\
\hline (2)-M 2 & -2347.364986 & 1.09 & 0.040 \\
\hline (2)-M 4 & -2347.364752 & 1.24 & 0.031 \\
\hline (2)-P 2 & -2347.364347 & 1.49 & 0.020 \\
\hline (2)-P 5 & -2347.363409 & 2.08 & 0.007 \\
\hline (2)-M5 & -2347.363324 & 2.13 & 0.007 \\
\hline
\end{tabular}




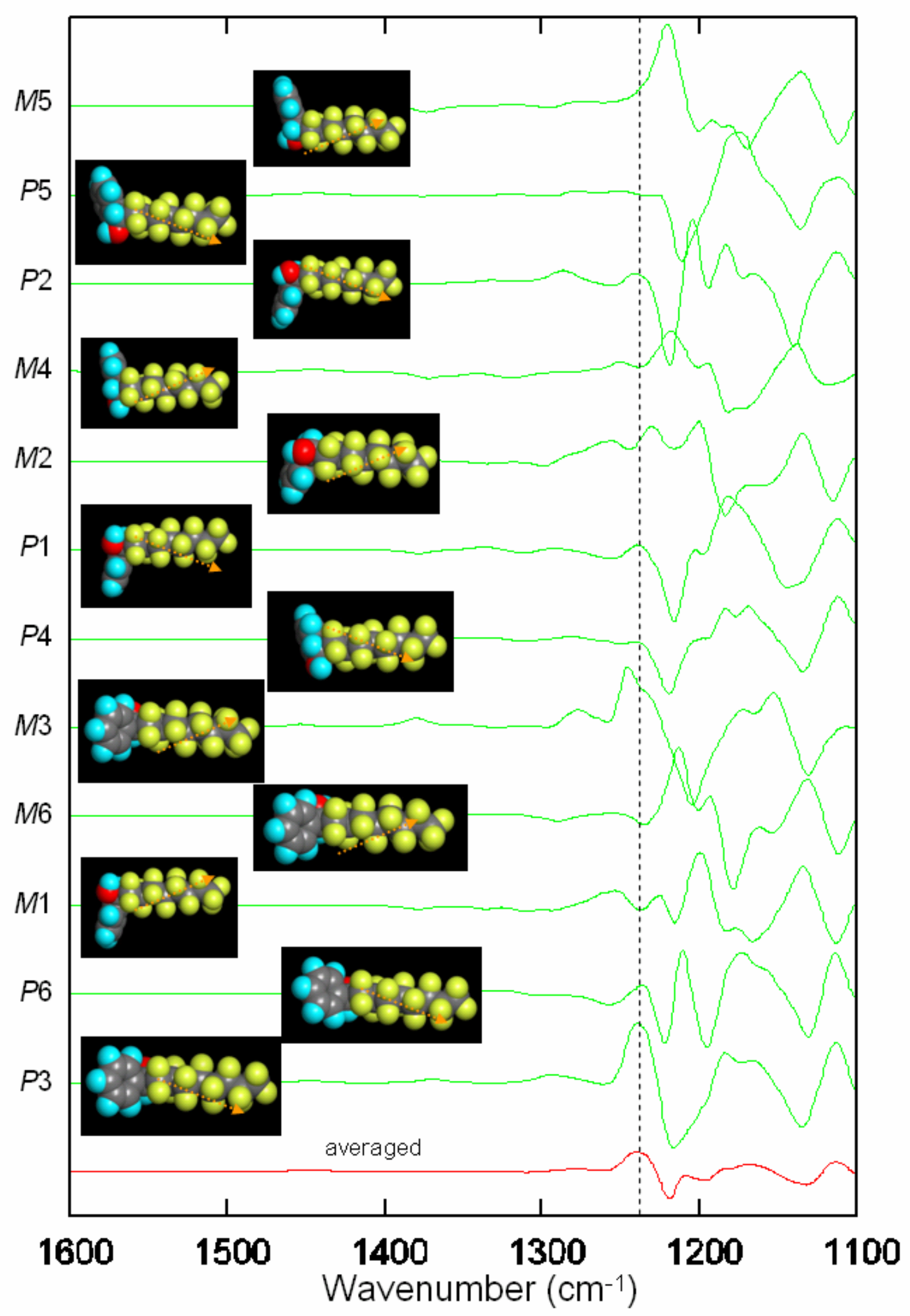

Figure S4. Calculated VCD spectra for $(R)$-2. Green lines and a red line indicate the VCD spectra for each conformer and a weighted averaged VCD spectrum, respectively. 


\section{Cartesian coordinates of all optimized geometries.}

\begin{tabular}{|c|c|c|c|c|c|}
\hline \multirow{2}{*}{$\begin{array}{l}\text { Center } \\
\text { Number }\end{array}$} & \multirow{2}{*}{$\begin{array}{l}\text { Atomic } \\
\text { Number }\end{array}$} & \multirow{2}{*}{$\begin{array}{l}\text { Atomic } \\
\text { Type }\end{array}$} & \multicolumn{3}{|c|}{ Coordinates (Angstroms) } \\
\hline & & & $x$ & $Y$ & Z \\
\hline 1 & 6 & $\odot$ & $\odot .00000 \odot$ & $\odot .00000 \odot$ & $\odot .00000 \odot$ \\
\hline 2 & 6 & $\odot$ & $\odot .0 \odot \odot \odot \odot \odot$ & $\odot .00 \odot \odot \odot \odot$ & 1.552200 \\
\hline 3 & 9 & $\odot$ & 1.281901 & $\odot .0 \odot \odot \odot \odot \odot$ & 1.962293 \\
\hline 4 & 9 & $\odot$ & $\odot .837104$ & -0.945841 & -0.429494 \\
\hline 5 & 9 & $\odot$ & -1.219587 & -0.260344 & $-\odot .469735$ \\
\hline 6 & 9 & $\odot$ & $\odot .408075$ & 1.183123 & -0.466680 \\
\hline 7 & 6 & $\odot$ & $-\odot .723069$ & 1.219726 & 2.190043 \\
\hline 8 & 9 & $\odot$ & $\odot .080519$ & 2.295000 & 2.061651 \\
\hline 9 & 9 & $\odot$ & $-\odot .600754$ & -1.137506 & 1.959035 \\
\hline 10 & 6 & $\odot$ & -1.067887 & 1.018702 & 3.696773 \\
\hline 11 & 9 & $\odot$ & -2.143076 & $\odot .209626$ & 3.775511 \\
\hline 12 & 9 & $\odot$ & -1.872721 & 1.441079 & 1.521101 \\
\hline 13 & 6 & $\odot$ & -1.382828 & 2.353627 & 4.439160 \\
\hline 14 & 9 & $\odot$ & $-\odot .216580$ & 2.980512 & 4.689434 \\
\hline 15 & 9 & $\odot$ & $-\odot .020695$ & 0.433339 & 4.310586 \\
\hline 16 & 6 & $\odot$ & -2.143198 & 2.144500 & 5.783648 \\
\hline 17 & 9 & $\odot$ & -3.433919 & 1.877302 & 5.499823 \\
\hline 18 & 9 & $\odot$ & -2.146061 & 3.128079 & 3.642753 \\
\hline 19 & 6 & $\odot$ & -2.083541 & 3.379056 & 6.727061 \\
\hline 20 & 9 & $\odot$ & $-\odot .863458$ & 3.426258 & 7.293054 \\
\hline 21 & 9 & $\odot$ & -1.604300 & 1.094546 & 6.435851 \\
\hline 22 & 6 & $\odot$ & -3.134292 & 3.358981 & 7.869222 \\
\hline 23 & 9 & $\odot$ & -4.357670 & 3.572991 & 7. 386141 \\
\hline 24 & 9 & $\odot$ & -2.287223 & 4.501505 & 6.006484 \\
\hline 25 & 9 & $\odot$ & -2.840573 & 4.326118 & 8.740122 \\
\hline 26 & 9 & $\odot$ & -3.111366 & 2.183892 & 8.503837 \\
\hline
\end{tabular}

(4)-M2

\begin{tabular}{|c|c|c|c|c|c|}
\hline \multirow{2}{*}{$\begin{array}{l}\text { Center } \\
\text { Number }\end{array}$} & \multirow{2}{*}{$\begin{array}{l}\text { Atomic } \\
\text { Number }\end{array}$} & \multirow{2}{*}{$\begin{array}{c}\text { Atomic } \\
\text { Type }\end{array}$} & \multicolumn{3}{|c|}{ Coordinates (Angstroms) } \\
\hline & & & $\mathrm{x}$ & $Y$ & Z \\
\hline 1 & 6 & $\odot$ & $\odot . ๑ \odot \odot \odot \odot \odot$ & $\odot . \odot \odot \odot \odot \odot \odot$ & $\odot . \odot \odot \odot \odot \odot \odot$ \\
\hline 2 & 6 & 0 & $\odot .000000$ & $\odot .000000$ & 1.552400 \\
\hline 3 & 9 & 0 & 1.285591 & $\odot .000000$ & 1.959473 \\
\hline 4 & 9 & $\odot$ & $\odot .820831$ & $-\odot .962430$ & -0.424957 \\
\hline 5 & 9 & $\odot$ & -1.223391 & $-\odot .240020$ & $-\odot .47010 \odot$ \\
\hline 6 & 9 & $\odot$ & $\odot .429161$ & 1.173373 & $-\odot .470363$ \\
\hline 7 & 6 & $\odot$ & $-\odot .716195$ & 1.213919 & 2.195910 \\
\hline 8 & 9 & $\odot$ & $\bullet .031786$ & 2.313070 & 1.989767 \\
\hline 9 & 9 & $\odot$ & $-\odot .597019$ & -1.139086 & 1.956659 \\
\hline 10 & 6 & $\odot$ & -1.044963 & 1.117572 & 3.721823 \\
\hline 11 & 9 & $\odot$ & -1.579721 & 2.313173 & 4.053549 \\
\hline 12 & 9 & $\odot$ & -1.908562 & 1.374994 & 1.579508 \\
\hline 13 & 6 & $\odot$ & $\odot .146798$ & $\odot .834304$ & 4.677731 \\
\hline 14 & 9 & $\odot$ & $\odot .474277$ & -0.470212 & 4.559262 \\
\hline
\end{tabular}




\begin{tabular}{|c|c|c|c|c|c|}
\hline 15 & 9 & $\odot$ & -1.972577 & ๑.158541 & 3.890782 \\
\hline 16 & 6 & $\odot$ & -0.169816 & 1.140717 & 6.173591 \\
\hline 17 & 9 & $\odot$ & -0.118423 & 2.476776 & 6.348199 \\
\hline 18 & 9 & $\odot$ & 1.196362 & 1.594486 & 4.311482 \\
\hline 19 & 6 & $\odot$ & 0.822510 & ๑.467999 & 7.163926 \\
\hline 20 & 9 & $\odot$ & 0.515561 & -0.839203 & 7.254391 \\
\hline 21 & 9 & $\odot$ & -1.410276 & $\odot .7 \odot 2259$ & 6.465667 \\
\hline 22 & 6 & $\odot$ & 0.791473 & 1.061762 & 8.597951 \\
\hline 23 & 9 & $\odot$ & 1.338231 & 2.276803 & 8.614929 \\
\hline 24 & 9 & $\odot$ & 2.080963 & 0.601222 & 6.696070 \\
\hline 25 & 9 & $\odot$ & 1.490365 & $\odot .264012$ & 9.407416 \\
\hline 26 & 9 & $\odot$ & -0.464868 & 1.138270 & 9.044994 \\
\hline
\end{tabular}

(4) $-M 3$

\begin{tabular}{|c|c|c|c|c|c|}
\hline \multirow{2}{*}{$\begin{array}{l}\text { Center } \\
\text { Number }\end{array}$} & \multirow{2}{*}{$\begin{array}{l}\text { Atomic } \\
\text { Number }\end{array}$} & \multirow{2}{*}{$\begin{array}{c}\text { Atomic } \\
\text { Type }\end{array}$} & \multicolumn{3}{|c|}{ Coordinates (Angstroms) } \\
\hline & & & $x$ & $Y$ & Z \\
\hline 1 & 6 & 0 & 0.000000 & 0.000000 & 0.000000 \\
\hline 2 & 6 & $\odot$ & $\odot . \odot \odot \odot \odot \odot \odot$ & $\odot . \diamond \odot \odot \odot \odot \odot$ & 1.547400 \\
\hline 3 & 9 & $\odot$ & 1.288939 & $\odot . \odot \odot \odot \odot \odot \odot$ & 1.947133 \\
\hline 4 & 9 & 0 & 0.794936 & $-\odot .980963$ & -0.429585 \\
\hline 5 & 9 & $\odot$ & -1.232840 & $-\odot .213170$ & $-\odot .466287$ \\
\hline 6 & 9 & $\odot$ & 0.452232 & 1.162914 & $-\odot .472731$ \\
\hline 7 & 6 & 0 & $-\odot .668852$ & 1.208133 & 2.271171 \\
\hline 8 & 9 & $\odot$ & $-\odot .6 \odot 2182$ & $\odot .930464$ & 3.591545 \\
\hline 9 & 9 & $\odot$ & $-\odot .587865$ & -1.137019 & 1.964038 \\
\hline 10 & 6 & $\odot$ & -2.148722 & 1.509150 & 1.905988 \\
\hline 11 & 9 & 0 & -2.161003 & 2.107559 & ๑.695031 \\
\hline 12 & 9 & $\odot$ & $\odot .066469$ & 2.305207 & 2.011987 \\
\hline 13 & 6 & $\odot$ & -2.866364 & 2.437169 & 2.933970 \\
\hline 14 & 9 & 0 & -3.189553 & 1.696729 & 4.012294 \\
\hline 15 & 9 & $\odot$ & -2.832163 & $\odot .350786$ & 1.839422 \\
\hline 16 & 6 & $\odot$ & -4.160573 & 3.094787 & 2.365329 \\
\hline 17 & 9 & 0 & -3.795525 & 4.108232 & 1.554247 \\
\hline 18 & 9 & $\odot$ & -2.024235 & 3.421742 & 3.303271 \\
\hline 19 & 6 & 0 & -5.107573 & 3.650300 & 3.466097 \\
\hline 20 & 9 & 0 & -5.746372 & 2.615618 & 4.042764 \\
\hline 21 & 9 & $\odot$ & -4.844489 & 2.177965 & 1. 651271 \\
\hline 22 & 6 & $\odot$ & -6.184972 & 4.637123 & 2.941666 \\
\hline 23 & 9 & 0 & -5.630105 & 5.797519 & 2.592820 \\
\hline 24 & 9 & 0 & -4.377487 & 4.301133 & 4.395614 \\
\hline 25 & 9 & $\odot$ & -7.071859 & 4.859044 & 3.913266 \\
\hline 26 & 9 & $\odot$ & -6.819327 & 4.118423 & 1.886849 \\
\hline
\end{tabular}

(4)-M4

\begin{tabular}{|c|c|c|c|c|c|}
\hline \multirow{2}{*}{$\begin{array}{l}\text { Center } \\
\text { Number }\end{array}$} & \multirow{2}{*}{$\begin{array}{l}\text { Atomic } \\
\text { Number }\end{array}$} & \multirow{2}{*}{$\begin{array}{l}\text { Atomic } \\
\text { Type }\end{array}$} & \multicolumn{3}{|c|}{ Coordinates (Angstroms) } \\
\hline & & & $x$ & Y & z \\
\hline 1 & 6 & 0 & $\odot .0000 \bullet \odot$ & 0.000000 & $\odot .000000$ \\
\hline 2 & 6 & $\odot$ & $\odot . \diamond \odot \odot \odot \odot \odot$ & $\odot . \diamond \odot \odot \odot \odot \odot$ & 1.552200 \\
\hline
\end{tabular}




\begin{tabular}{|c|c|c|c|c|c|}
\hline 3 & 9 & $\odot$ & 1.282568 & $\odot . \diamond \odot \odot \odot \odot \odot$ & 1.961520 \\
\hline 4 & 9 & $\odot$ & 0.828453 & $-\odot .954372$ & -0.428014 \\
\hline 5 & 9 & $\odot$ & -1.221831 & $-\odot .249251$ & $-\odot .47 \odot 2 \odot 5$ \\
\hline 6 & 9 & $\odot$ & 0.419333 & 1.178275 & -0.468104 \\
\hline 7 & 6 & $\odot$ & -0.719477 & 1.220942 & 2.191775 \\
\hline 8 & 9 & $\odot$ & 0.076754 & 2.296067 & 2.036015 \\
\hline 9 & 9 & $\odot$ & -0.599363 & -1.138237 & 1.958047 \\
\hline 10 & 6 & $\odot$ & -1.054992 & 1.020783 & 3.700512 \\
\hline 11 & 9 & $\odot$ & -2.120888 & $\odot .203714$ & 3.767454 \\
\hline 12 & 9 & $\odot$ & -1.880111 & 1.434160 & 1.538119 \\
\hline 13 & 6 & $\odot$ & -1.423772 & 2.336722 & 4.476953 \\
\hline 14 & 9 & $\odot$ & -1.974527 & 3.216847 & 3.612361 \\
\hline 15 & 9 & $\odot$ & -0.012979 & $\odot .423236$ & 4.319074 \\
\hline 16 & 6 & $\odot$ & -0.234737 & 3.013899 & 5.222767 \\
\hline 17 & 9 & $\odot$ & ๑.026961 & 2.283384 & 6.326367 \\
\hline 18 & 9 & $\odot$ & -2.342633 & 2.011147 & 5.403189 \\
\hline 19 & 6 & $\odot$ & -0.528533 & 4.479904 & 5.649284 \\
\hline 20 & 9 & $\odot$ & -0.448658 & 5.264135 & 4.557897 \\
\hline 21 & 9 & $\odot$ & ๑.856843 & 3.025281 & 4.433804 \\
\hline 22 & 6 & $\odot$ & $\odot .453823$ & 5.045439 & 6.709695 \\
\hline 23 & 9 & $\odot$ & $\odot .247682$ & 4.471529 & 7.894694 \\
\hline 24 & 9 & $\odot$ & -1.772107 & 4.557165 & 6.166087 \\
\hline 25 & 9 & $\odot$ & ๑.248166 & 6.358650 & 6.826050 \\
\hline 26 & 9 & $\odot$ & 1.718486 & 4.837217 & 6.334419 \\
\hline
\end{tabular}

(4)-P1

\begin{tabular}{|c|c|c|c|c|c|}
\hline \multirow{2}{*}{$\begin{array}{l}\text { Center } \\
\text { Number }\end{array}$} & \multirow{2}{*}{$\begin{array}{l}\text { Atomic } \\
\text { Number }\end{array}$} & \multirow{2}{*}{$\begin{array}{l}\text { Atomic } \\
\text { Type }\end{array}$} & \multicolumn{3}{|c|}{ Coordinates (Angstroms) } \\
\hline & & & $x$ & Y & Z \\
\hline 1 & 6 & 0 & $\odot .000000$ & 0.000000 & 0.000000 \\
\hline 2 & 6 & $\odot$ & $\odot . \odot \odot \odot \odot \odot \odot$ & $\odot .000000$ & 1.552100 \\
\hline 3 & 9 & $\odot$ & 1.286496 & 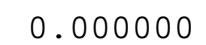 & 1.958965 \\
\hline 4 & 9 & $\odot$ & $\odot .445467$ & -1.182014 & $-\odot .429526$ \\
\hline 5 & 9 & 0 & -1.236755 & ๑.191681 & -0.466680 \\
\hline 6 & 9 & 0 & $\odot .799650$ & $\odot .956710$ & -0.469917 \\
\hline 7 & 6 & $\odot$ & -0.741073 & 1.208848 & 2.190232 \\
\hline 8 & 9 & 0 & -0.400025 & 2.328914 & 1.521614 \\
\hline 9 & 9 & 0 & -0.598653 & -1.133528 & 1.962193 \\
\hline 10 & 6 & $\odot$ & $-\odot .402017$ & 1.419847 & 3.696802 \\
\hline 11 & 9 & $\odot$ & -0.373015 & $\odot .220462$ & 4.310529 \\
\hline 12 & 9 & $\odot$ & -2.067045 & 1.000231 & 2.061939 \\
\hline 13 & 6 & $\odot$ & -1.435749 & 2.321330 & 4.439358 \\
\hline 14 & 9 & $\odot$ & -1.764355 & 3.357992 & 3.643171 \\
\hline 15 & 9 & $\odot$ & $\odot .815206$ & 1.993202 & 3.775523 \\
\hline 16 & 6 & $\odot$ & $-\odot .896057$ & 2.895824 & 5.783949 \\
\hline 17 & 9 & $\odot$ & -0.219033 & 1.929156 & 6.436157 \\
\hline 18 & 9 & $\Theta$ & -2.534696 & 1.582456 & 4.689174 \\
\hline 19 & 6 & $\odot$ & -2.015613 & 3.419357 & 6.727464 \\
\hline 20 & 9 & 0 & -2.913305 & 4.123361 & 6.006952 \\
\hline 21 & 9 & $\Theta$ & $-\odot .057048$ & 3.912611 & 5.500417 \\
\hline 22 & 6 & $\odot$ & -1.507214 & 4.339184 & 7.869731 \\
\hline 23 & 9 & $\odot$ & $-\odot .478644$ & 3.770414 & 8.504059 \\
\hline
\end{tabular}




$\begin{array}{llllll}24 & 9 & \odot & -2.626919 & 2.362393 & 7.293422 \\ 25 & 9 & \odot & -2.499483 & 4.530874 & 8.740637 \\ 26 & 9 & \odot & -1.125565 & 5.521081 & 7.386734\end{array}$

(4) $-P 2$

\begin{tabular}{|c|c|c|c|c|c|}
\hline \multirow{2}{*}{$\begin{array}{l}\text { Center } \\
\text { Number }\end{array}$} & \multirow{2}{*}{$\begin{array}{l}\text { Atomic } \\
\text { Number }\end{array}$} & \multirow{2}{*}{$\begin{array}{l}\text { Atomic } \\
\text { Type }\end{array}$} & \multicolumn{3}{|c|}{ Coordinates (Angstroms) } \\
\hline & & & $X$ & $\mathrm{Y}$ & Z \\
\hline- & & & 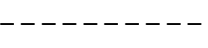 & - - - - - - & - \\
\hline 1 & 6 & $\odot$ & $\odot . ๑ \odot \odot \odot \odot \odot$ & $\odot .0 \odot \odot \odot \odot \odot$ & $\odot .0 \odot \odot \odot \odot \odot$ \\
\hline 2 & 6 & $\odot$ & $\odot . ๑ \odot \odot \odot \odot \odot$ & $\odot . \odot \odot \odot \odot \odot \odot$ & 1.552800 \\
\hline 3 & 9 & $\odot$ & 1.286112 & $\odot .0 \odot \odot \odot \odot \odot$ & 1.958556 \\
\hline 4 & 9 & $\odot$ & $\odot .429918$ & -1.187636 & -0.429241 \\
\hline 5 & 9 & $\odot$ & -1.233443 & ๑. 208621 & -0.468462 \\
\hline 6 & 9 & $\odot$ & 0.812864 & $\odot .946711$ & -0.467775 \\
\hline 7 & 6 & $\odot$ & -0.746194 & 1.208663 & 2.187007 \\
\hline 8 & 9 & $\odot$ & $-\odot .415981$ & 2.328078 & 1.506714 \\
\hline 9 & 9 & 0 & -0.597561 & -1.133376 & 1.963920 \\
\hline 10 & 6 & $\odot$ & -0.403587 & 1.437580 & 3.684451 \\
\hline 11 & 9 & $\odot$ & -0.425688 & ๑. 240290 & 4.310781 \\
\hline 12 & 9 & $\odot$ & -2.069685 & ๑. 988777 & 2.062473 \\
\hline 13 & 6 & $\odot$ & -1.375873 & 2.352809 & 4.499919 \\
\hline 14 & 9 & $\odot$ & -2.558773 & 1.722479 & 4.606600 \\
\hline 15 & 9 & $\odot$ & 0.834616 & 1.954968 & 3.770367 \\
\hline 16 & 6 & $\odot$ & -1.620595 & 3.783759 & 3.948002 \\
\hline 17 & 9 & $\odot$ & -0.448011 & 4.312032 & 3.546514 \\
\hline 18 & 9 & $\odot$ & -0.822117 & 2.462521 & 5.727763 \\
\hline 19 & 6 & $\odot$ & -2.250849 & 4.755118 & 4.986964 \\
\hline 20 & 9 & $\odot$ & -3.225587 & 4.118215 & 5.667576 \\
\hline 21 & 9 & $\odot$ & -2.450332 & 3.685736 & 2.886339 \\
\hline 22 & 6 & $\odot$ & -2.875321 & 6.032047 & 4.362172 \\
\hline 23 & 9 & $\odot$ & -2.021808 & 6.600128 & 3.505924 \\
\hline 24 & 9 & $\odot$ & -1.289428 & 5.144534 & 5.844090 \\
\hline 25 & 9 & $\odot$ & -3.148056 & 6.898587 & 5.339044 \\
\hline 26 & 9 & $\odot$ & $-4.0 \odot 6 \odot 52$ & 5.735166 & 3.722537 \\
\hline
\end{tabular}

(4)-P3

\begin{tabular}{|c|c|c|c|c|c|}
\hline \multirow{2}{*}{$\begin{array}{l}\text { Center } \\
\text { Number }\end{array}$} & \multirow{2}{*}{$\begin{array}{l}\text { Atomic } \\
\text { Number }\end{array}$} & \multirow{2}{*}{$\begin{array}{l}\text { Atomic } \\
\text { Type }\end{array}$} & \multicolumn{3}{|c|}{ Coordinates (Angstroms) } \\
\hline & & & $x$ & $\mathrm{Y}$ & Z \\
\hline \multicolumn{6}{|c|}{ 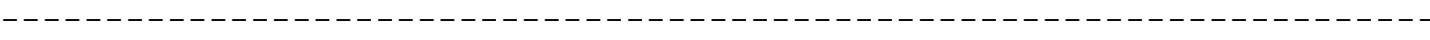 } \\
\hline 1 & 6 & $\odot$ & $\odot .0000 \odot \odot$ & $\odot .000000$ & $\odot .000000$ \\
\hline 2 & 6 & $\odot$ & $\odot .000000$ & $\odot .000000$ & 1.553300 \\
\hline 3 & 9 & $\odot$ & 1.286445 & $\odot .000000$ & 1.953983 \\
\hline 4 & 9 & $\odot$ & ○. 849177 & -0.936511 & -0.427164 \\
\hline 5 & 9 & $\odot$ & -1.214727 & -0.275756 & -0.472674 \\
\hline 6 & 9 & $\odot$ & ○. 396281 & 1.187112 & -0.466427 \\
\hline 7 & 6 & $\odot$ & -0.712889 & 1.226836 & 2.183561 \\
\hline 8 & 9 & $\odot$ & 0.117874 & 2.282603 & 2.100465 \\
\hline 9 & 9 & $\odot$ & $-\odot .598831$ & -1.137227 & 1.959773 \\
\hline 10 & 6 & $\odot$ & -1.213786 & 1.119620 & 3.662779 \\
\hline 11 & 9 & $\odot$ & -1.756549 & 2.325074 & 3.931274 \\
\hline
\end{tabular}




\begin{tabular}{|c|c|c|c|c|c|}
\hline 12 & 9 & $\odot$ & -1.824756 & 1.484235 & 1.457782 \\
\hline 13 & 6 & $\odot$ & -0.148963 & ๑. 812709 & 4.748505 \\
\hline 14 & 9 & $\odot$ & $\odot .464137$ & $-\odot .346073$ & 4.430916 \\
\hline 15 & 9 & $\odot$ & -2.175977 & $\odot .178495$ & 3.723516 \\
\hline 16 & 6 & $\odot$ & -0.729619 & $\odot .646509$ & 6.187710 \\
\hline 17 & 9 & $\odot$ & -1.635888 & 1.611629 & 6.432686 \\
\hline 18 & 9 & $\odot$ & 0.754200 & 1.813813 & 4.748739 \\
\hline 19 & 6 & $\odot$ & 0.357441 & 0.721753 & 7.298744 \\
\hline 20 & 9 & $\odot$ & 1.447000 & ๑. . 025878 & 6.914191 \\
\hline 21 & 9 & $\odot$ & -1.337930 & -0.555800 & 6.252609 \\
\hline 22 & 6 & $\odot$ & -0.097105 & ๑. 155878 & 8.671126 \\
\hline 23 & 9 & $\odot$ & -1.284644 & $\odot .660504$ & 9.015987 \\
\hline 24 & 9 & $\odot$ & ๑. 693442 & 2.013032 & 7.476747 \\
\hline 25 & 9 & $\odot$ & $\odot .802308$ & $\odot .497623$ & 9.595296 \\
\hline 26 & 9 & $\odot$ & -0.181520 & -1.173230 & 8.624550 \\
\hline
\end{tabular}

(4) $-P 4$

\begin{tabular}{|c|c|c|c|c|c|}
\hline \multirow{2}{*}{$\begin{array}{l}\text { Center } \\
\text { Number }\end{array}$} & \multirow{2}{*}{$\begin{array}{l}\text { Atomic } \\
\text { Number }\end{array}$} & \multirow{2}{*}{$\begin{array}{c}\text { Atomic } \\
\text { Type }\end{array}$} & \multicolumn{3}{|c|}{ Coordinates (Angstroms) } \\
\hline & & & $x$ & $\mathrm{Y}$ & Z \\
\hline & & & & - - - - - - - &.- \\
\hline 1 & 6 & $\odot$ & $\odot . \odot \odot \odot \odot \odot \odot$ & $\odot . \odot \odot \odot \odot \odot \odot$ & $\odot . \odot \odot \odot \odot \odot \odot$ \\
\hline 2 & 6 & $\odot$ & $\odot . \odot \odot \odot \odot \odot \odot$ & $\odot .0000 \odot \odot$ & 1.552000 \\
\hline 3 & 9 & $\odot$ & 1. 289652 & $\odot . \odot \odot \odot \odot \odot \odot$ & 1.948749 \\
\hline 4 & 9 & $\odot$ & 0.935710 & -0.850834 & -0.425619 \\
\hline 5 & 9 & $\odot$ & -1.181335 & -0.404462 & -0.468344 \\
\hline 6 & 9 & $\odot$ & $\odot .273248$ & 1.215463 & -0.478966 \\
\hline 7 & 6 & $\odot$ & -0.660143 & 1.194369 & 2.310314 \\
\hline 8 & 9 & $\odot$ & -0.498055 & ๑. 911916 & 3.619211 \\
\hline 9 & 9 & $\odot$ & -0.588157 & -1.140510 & 1.962520 \\
\hline 10 & 6 & $\odot$ & -2.167457 & 1. 449184 & 2.048849 \\
\hline 11 & 9 & $\odot$ & -2.343572 & 1.627176 & 0.722681 \\
\hline 12 & 9 & $\odot$ & 0.021422 & 2.318467 & 2.011122 \\
\hline 13 & 6 & $\odot$ & -2.735833 & 2.715054 & 2.765824 \\
\hline 14 & 9 & $\odot$ & -2.274847 & 2.769578 & 4.029139 \\
\hline 15 & 9 & $\odot$ & -2.856682 & 0.362982 & 2.451636 \\
\hline 16 & 6 & $\odot$ & -4.294510 & 2.733490 & 2.822058 \\
\hline 17 & 9 & $\odot$ & -4.788045 & 2.308179 & 1.641729 \\
\hline 18 & 9 & $\odot$ & -2.307763 & 3.800141 & 2.090415 \\
\hline 19 & 6 & $\odot$ & -4.885175 & 4.142721 & 3.109975 \\
\hline 20 & 9 & $\odot$ & -4.171226 & 4.734494 & 4.089848 \\
\hline 21 & 9 & $\odot$ & -4.687653 & 1.889476 & 3.797523 \\
\hline 22 & 6 & $\odot$ & -6.372009 & 4.132455 & 3.555559 \\
\hline 23 & 9 & $\odot$ & -7.105978 & 3.397310 & 2.715948 \\
\hline 24 & 9 & $\odot$ & -4.799562 & 4.879124 & 1.986544 \\
\hline 25 & 9 & $\odot$ & -6.830623 & 5.385330 & 3.547303 \\
\hline 26 & 9 & $\odot$ & -6.490071 & 3.639788 & 4.788102 \\
\hline
\end{tabular}

(2)-P3

Center Atomic Atomic Coordinates (Angstroms)




\begin{tabular}{|c|c|c|c|c|c|}
\hline Number & Number & Type & $x$ & Y & Z \\
\hline & & - & 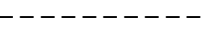 & 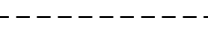 & -5 \\
\hline 1 & 6 & $\odot$ & 0.102098 & ๑. 372417 & -0.094327 \\
\hline 2 & 6 & 0 & ๑. 053095 & ๑. 327139 & 1.298451 \\
\hline 3 & 6 & 0 & 1.189124 & -0.021752 & 2.035227 \\
\hline 4 & 6 & $\odot$ & 2.375685 & -0.334495 & 1. 361881 \\
\hline 5 & 6 & $\odot$ & 2.422245 & -0.293853 & -0.030228 \\
\hline 6 & 6 & $\odot$ & 1.287426 & ○.061792 & -0.760715 \\
\hline 7 & 6 & $\odot$ & 1.126728 & -0.083378 & 3.548194 \\
\hline 8 & 6 & $\odot$ & 1.670256 & 1.208008 & 4.191548 \\
\hline 9 & 9 & $\odot$ & 2.945580 & 1.449240 & 3.807398 \\
\hline 10 & 8 & $\odot$ & 1.880907 & -1.142450 & 4.097038 \\
\hline 11 & 6 & $\odot$ & 1.633466 & 1.214485 & 5.755752 \\
\hline 12 & 9 & $\odot$ & 2.668395 & ๑. 491517 & 6.227155 \\
\hline 13 & 9 & $\odot$ & ๑. 896938 & 2.244151 & 3.756427 \\
\hline 14 & 9 & $\odot$ & ๑. 473993 & 0.636943 & 6.157843 \\
\hline 15 & 6 & $\odot$ & 1.697935 & 2.642744 & 6.400571 \\
\hline 16 & 9 & $\odot$ & ๑. 487556 & 3.229401 & 6.273503 \\
\hline 17 & 9 & $\odot$ & 2.615969 & 3.381867 & 5.744731 \\
\hline 18 & 6 & $\odot$ & 2.084451 & 2.631841 & 7.920908 \\
\hline 19 & 9 & $\odot$ & 3.412021 & 2.413175 & 8.022648 \\
\hline 20 & 9 & $\odot$ & 1.423556 & 1.629979 & 8.537057 \\
\hline 21 & 6 & $\odot$ & 1.740699 & 3.968462 & 8.667606 \\
\hline 22 & 9 & $\odot$ & ๑. 413515 & 3.993267 & 8.909411 \\
\hline 23 & 9 & $\odot$ & 2.063453 & 5.012278 & 7.876964 \\
\hline 24 & 6 & $\odot$ & 2.501900 & 4.138793 & 10.028228 \\
\hline 25 & 9 & $\odot$ & 3.773898 & 4.501850 & 9.761949 \\
\hline 26 & 9 & $\odot$ & 2.512167 & 2.958155 & 10.681582 \\
\hline 27 & 6 & $\odot$ & 1.866613 & 5.207565 & 10.978386 \\
\hline 28 & 9 & $\odot$ & ๑. 759293 & 4.684306 & 11.539585 \\
\hline 29 & 9 & $\odot$ & 1.524810 & 6.301314 & 10.264327 \\
\hline 30 & 6 & $\odot$ & 2.797146 & 5.684194 & 12.138889 \\
\hline 31 & 9 & $\odot$ & 3.801247 & 6.429820 & 11.673906 \\
\hline 32 & 9 & $\odot$ & 3. 299757 & 4.638627 & 12.803621 \\
\hline 33 & 9 & $\odot$ & 2.075441 & 6.424570 & 12.986329 \\
\hline 34 & 1 & $\odot$ & ๑. 074201 & -0.141403 & 3.858596 \\
\hline 35 & 1 & $\odot$ & 1.583267 & -1.965524 & 3.691795 \\
\hline 36 & 1 & $\odot$ & 3.257259 & -0.609588 & 1.932797 \\
\hline 37 & 1 & $\odot$ & 3.347326 & -0.536926 & -0.545427 \\
\hline 38 & 1 & $\odot$ & 1.325763 & ๑. 092873 & -1.846035 \\
\hline 39 & 1 & $\odot$ & -0.786306 & 0.644014 & -0.657413 \\
\hline 40 & 1 & $\odot$ & -0.873678 & 0.565333 & 1.815261 \\
\hline
\end{tabular}

(2)-P6

\begin{tabular}{cccccc} 
Center & Atomic & Atomic & \multicolumn{3}{c}{ Coordinates } \\
Number & Number & Type & $X$ & $Y$ & (Angstroms ) \\
1 & 6 & 0 & 0.011693 & 0.042216 & -0.013735 \\
2 & 6 & 0 & -0.004299 & 0.043419 & 1.380467 \\
3 & 6 & 0 & 1.193044 & 0.003378 & 2.100295 \\
4 & 6 & 0 & 2.409263 & -0.046928 & 1.412199 \\
5 & 6 & 0 & 2.423247 & -0.052664 & 0.018863
\end{tabular}




\begin{tabular}{|c|c|c|c|c|c|}
\hline 6 & 6 & $\odot$ & 1.226412 & $-\odot .004855$ & -0.697125 \\
\hline 7 & 6 & $\odot$ & 1.170176 & 0.012867 & 3.612726 \\
\hline 8 & 6 & $\odot$ & 1.248670 & 1.469604 & 4.144584 \\
\hline 9 & 9 & $\odot$ & 2.358042 & 2.096206 & 3.690610 \\
\hline 10 & 8 & $\odot$ & 2.247792 & $-\odot .757497$ & 4.094278 \\
\hline 11 & 6 & $\Theta$ & 1.248161 & 1.579930 & 5.702723 \\
\hline 12 & 9 & $\odot$ & 2.472670 & 1.231407 & 6.164499 \\
\hline 13 & 9 & $\odot$ & ๑.157783 & 2.153468 & 3.692583 \\
\hline 14 & 9 & $\odot$ & ๑.348785 & ๑.681768 & 6.188314 \\
\hline 15 & 6 & $\odot$ & 0.877903 & 2.989135 & 6.277395 \\
\hline 16 & 9 & $\odot$ & $-\odot .459642$ & 3.148207 & 6.184899 \\
\hline 17 & 9 & $\odot$ & 1.488298 & 3.936832 & 5.539437 \\
\hline 18 & 6 & $\odot$ & 1.300542 & 3.192199 & 7.774387 \\
\hline 19 & 9 & $\odot$ & 2.632255 & 3.403509 & 7.815246 \\
\hline 20 & 9 & $\odot$ & 1.010601 & 2.070539 & 8.467274 \\
\hline 21 & 6 & $\odot$ & 0.578892 & 4.396136 & 8.475518 \\
\hline 22 & 9 & $\odot$ & $-\odot .678596$ & 4.017000 & 8.783492 \\
\hline 23 & 9 & $\odot$ & 0.528292 & 5.437318 & 7.620363 \\
\hline 24 & 6 & $\odot$ & 1.294854 & 4.877351 & 9.785260 \\
\hline 25 & 9 & $\odot$ & 2.378980 & $5.60 \odot 234$ & 9.435848 \\
\hline 26 & 9 & $\odot$ & 1.697024 & 3.799194 & 10.490374 \\
\hline 27 & 6 & $\odot$ & ๑. 389507 & 5.750681 & 10.715948 \\
\hline 28 & 9 & $\Theta$ & $-\odot .478328$ & 4.943281 & 11.355990 \\
\hline 29 & 9 & $\odot$ & $-\odot .301457$ & 6.638193 & 9.969292 \\
\hline 30 & 6 & $\Theta$ & 1.162698 & 6.561765 & 11.804171 \\
\hline 31 & 9 & $\odot$ & 1.868443 & 7.551345 & 11.253964 \\
\hline 32 & 9 & $\odot$ & 1.987742 & 5.764954 & 12.491325 \\
\hline 33 & 9 & $\Theta$ & 0.274399 & 7.092179 & 12.650263 \\
\hline 34 & 1 & $\odot$ & 0.205173 & -0.380832 & 3.956041 \\
\hline 35 & 1 & $\odot$ & 2.124117 & $-\odot .954442$ & 5.030056 \\
\hline 36 & 1 & $\odot$ & 3.337930 & $-\odot .093788$ & 1. 970901 \\
\hline 37 & 1 & $\odot$ & 3.371976 & $-\odot .095082$ & -0.508801 \\
\hline 38 & 1 & $\odot$ & 1.240157 & -๑.๑०९861 & -1.783517 \\
\hline 39 & 1 & $\odot$ & $-\odot .924638$ & 0.071142 & -0.563786 \\
\hline 40 & 1 & $\odot$ & $-\odot .954049$ & $\odot .074202$ & 1.908766 \\
\hline
\end{tabular}

(2)-M1

\begin{tabular}{rrrrrr} 
Number & $\begin{array}{c}\text { Atomic } \\
\text { Number }\end{array}$ & $\begin{array}{c}\text { Atomic } \\
\text { Type }\end{array}$ & \multicolumn{2}{c}{ Coordinates } & \multicolumn{2}{c}{ (Angstroms) } \\
1 & 6 & 0 & .080481 & .236245 & -.037201 \\
2 & 6 & 0 & .031304 & .120784 & 1.356238 \\
3 & 6 & 0 & 1.222793 & .031142 & 2.080586 \\
4 & 6 & 0 & 2.451862 & .037316 & 1.421734 \\
5 & 6 & 0 & 2.496520 & .143162 & .032398 \\
6 & 6 & 0 & 1.308854 & .246983 & -.693874 \\
7 & 6 & 0 & -1.294545 & .122302 & 2.079708 \\
8 & 8 & 0 & -2.107949 & 1.140621 & 1.538527 \\
9 & 6 & 0 & -2.079870 & -1.210766 & 1.972468 \\
10 & 6 & 0 & -1.404668 & -2.452042 & 2.629320 \\
11 & 9 & 0 & -.409810 & -2.877219 & 1.822012 \\
12 & 9 & 0 & -2.358105 & -1.510684 & .683820
\end{tabular}




$\begin{array}{rrrrrr}13 & 9 & 0 & -3.283089 & -1.017433 & 2.611933 \\ 14 & 6 & 0 & -2.365923 & -3.657185 & 2.916601 \\ 15 & 9 & 0 & -3.101088 & -3.365135 & 4.011364 \\ 16 & 9 & 0 & -.866654 & -2.066296 & 3.814658 \\ 17 & 6 & 0 & -1.618937 & -5.013623 & 3.168159 \\ 18 & 9 & 0 & -1.200427 & -5.496281 & 1.980009 \\ 19 & 9 & 0 & -3.191821 & -3.824342 & 1.864448 \\ 20 & 6 & 0 & -2.509207 & -6.100807 & 3.866228 \\ 21 & 9 & 0 & -2.605356 & -5.794841 & 5.176698 \\ 22 & 9 & 0 & -.546168 & -4.785319 & 3.954170 \\ 23 & 6 & 0 & -1.942769 & -7.556828 & 3.731005 \\ 24 & 9 & 0 & -2.199350 & -7.998860 & 2.482343 \\ 25 & 9 & 0 & -3.740917 & -6.079419 & 3.317275 \\ 26 & 6 & 0 & -2.554270 & -8.569217 & 4.755738 \\ 27 & 9 & 0 & -2.002947 & -8.347288 & 5.964708 \\ 28 & 9 & 0 & -.606837 & -7.531431 & 3.919714 \\ 29 & 6 & 0 & -2.321231 & -10.073454 & 4.405781 \\ 30 & 9 & 0 & -3.034485 & -10.434114 & 3.337481 \\ 31 & 9 & 0 & -3.888251 & -8.378085 & 4.837183 \\ 32 & 9 & 0 & -2.709120 & -10.817009 & 5.446567 \\ 33 & 9 & 0 & -1.026780 & -10.309071 & 4.168040 \\ 34 & 1 & 0 & -1.119970 & .283492 & 3.151623 \\ 35 & 1 & 0 & -2.945359 & 1.161829 & 2.019693 \\ 36 & 1 & 0 & 1.193503 & -.042066 & 3.165032 \\ 37 & 1 & 0 & 3.371917 & -.032819 & 1.995016 \\ 38 & 1 & 3.452977 & .151979 & -.482925 \\ 39 & 1 & 1.338954 & .339053 & -1.775971 \\ 40 & 1 & -.842350 & .331709 & -.599429 \\ --- & - & 0 & 0 & ----------------------\end{array}$

(2)-M6

\begin{tabular}{|c|c|c|c|c|c|}
\hline \multirow{2}{*}{$\begin{array}{l}\text { Center } \\
\text { Number }\end{array}$} & Atomic & Atomic & \multicolumn{3}{|c|}{ Coordinates (Angstroms) } \\
\hline & Number & Type & $\mathrm{x}$ & Y & $\mathrm{Z}$ \\
\hline \multicolumn{6}{|c|}{ 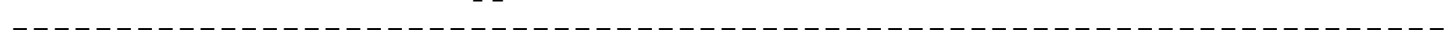 } \\
\hline 1 & 6 & $\odot$ & -.058521 & -.019733 & -.019463 \\
\hline 2 & 6 & $\odot$ & .107361 & -.084607 & 1.366343 \\
\hline 3 & 6 & $\odot$ & 1.396976 & - . 108602 & 1.905969 \\
\hline 4 & 6 & $\odot$ & 2.506552 & - .062912 & 1.063818 \\
\hline 5 & 6 & $\odot$ & 2.337439 & .008678 & -.319473 \\
\hline 6 & 6 & $\odot$ & 1.052130 & .029054 & -.860521 \\
\hline 7 & 6 & $\odot$ & -1.101554 & -.132969 & 2.275058 \\
\hline 8 & 8 & $\odot$ & - . 811709 & -.978255 & 3.362328 \\
\hline 9 & 6 & $\odot$ & -1.475856 & 1.298543 & 2.759312 \\
\hline 10 & 6 & 0 & -2.890680 & 1.394428 & 3.412585 \\
\hline 11 & 9 & 0 & -3.807291 & 1.198343 & 2.435632 \\
\hline 12 & 9 & $\odot$ & -1.491529 & 2.140245 & 1.692089 \\
\hline 13 & 9 & $\odot$ & -.562610 & 1.757715 & 3.649823 \\
\hline 14 & 6 & $\odot$ & -3.209216 & 2.735019 & 4.157450 \\
\hline 15 & 9 & $\odot$ & -2.570246 & 2.714253 & 5.346625 \\
\hline 16 & 9 & $\odot$ & -3.031791 & .383981 & 4.317302 \\
\hline 17 & 6 & $\odot$ & -4.739240 & 2.968482 & 4.414631 \\
\hline 18 & 9 & $\odot$ & -5.309221 & 3. 354551 & 3. 254162 \\
\hline 19 & 9 & $\odot$ & -2.747307 & 3.768177 & 3.427711 \\
\hline
\end{tabular}




\begin{tabular}{|c|c|c|c|c|c|}
\hline 20 & 6 & $\odot$ & -5.036292 & 4.053837 & 5.507689 \\
\hline 21 & 9 & $\odot$ & -4.813768 & 3.506335 & 6.720269 \\
\hline 22 & 9 & $\odot$ & -5.299341 & 1.807725 & 4.815199 \\
\hline 23 & 6 & $\odot$ & -6.505448 & 4.601021 & 5.464065 \\
\hline 24 & 9 & $\odot$ & -6.605611 & 5.467992 & 4.435275 \\
\hline 25 & 9 & $\odot$ & -4.197231 & 5.094632 & 5.333250 \\
\hline 26 & 6 & $\odot$ & -6.947008 & 5.328334 & 6.777179 \\
\hline 27 & 9 & $\odot$ & -7.200099 & 4.400877 & 7.720799 \\
\hline 28 & 9 & $\odot$ & -7.354068 & 3.573066 & 5.253031 \\
\hline 29 & 6 & $\odot$ & -8.227274 & 6.211845 & 6.636007 \\
\hline 30 & 9 & $\odot$ & -7.985605 & 7.292389 & 5.891693 \\
\hline 31 & 9 & $\odot$ & -5.949766 & 6.132131 & 7.203821 \\
\hline 32 & 9 & $\odot$ & -8.603971 & 6.610301 & 7.854796 \\
\hline 33 & 9 & $\odot$ & -9.225571 & 5.514262 & 6.084024 \\
\hline 34 & 1 & $\odot$ & -1.963688 & -.493764 & 1.695224 \\
\hline 35 & 1 & $\odot$ & -1.598065 & -1.087299 & 3.909442 \\
\hline 36 & 1 & $\odot$ & 1.524715 & -.177349 & 2.981089 \\
\hline 37 & 1 & $\odot$ & 3.505764 & -.085193 & 1.489750 \\
\hline 38 & 1 & $\odot$ & 3.204228 & .042923 & -.973688 \\
\hline 39 & 1 & $\odot$ & .912659 & .076848 & -1.936813 \\
\hline 40 & 1 & $\odot$ & -1.058895 & - . .007739 & -.445446 \\
\hline
\end{tabular}

(2)-M3

\begin{tabular}{|c|c|c|c|c|c|}
\hline \multirow{2}{*}{$\begin{array}{l}\text { Center } \\
\text { Number }\end{array}$} & \multirow{2}{*}{$\begin{array}{l}\text { Atomic } \\
\text { Number }\end{array}$} & \multirow{2}{*}{$\begin{array}{c}\text { Atomic } \\
\text { Type }\end{array}$} & \multicolumn{3}{|c|}{ Coordinates (Angstroms) } \\
\hline & & & $x$ & Y & Z \\
\hline & & & -------- & -------- & ------- \\
\hline 1 & 6 & $\odot$ & -0.020780 & -0.069691 & -0.016870 \\
\hline 2 & 6 & $\odot$ & -0.000484 & -0.048947 & 1.377162 \\
\hline 3 & 6 & $\odot$ & 1.214737 & -0.000313 & 2.066479 \\
\hline 4 & 6 & $\odot$ & 2.414122 & 0.019403 & 1.344637 \\
\hline 5 & 6 & $\odot$ & 2.392844 & -0.003837 & -0.048578 \\
\hline 6 & 6 & $\odot$ & 1.176592 & -0.046422 & -0.731858 \\
\hline 7 & 6 & $\odot$ & 1.232504 & 0.000460 & 3.582923 \\
\hline 8 & 6 & $\odot$ & 1.472180 & 1.422306 & 4.139828 \\
\hline 9 & 9 & $\odot$ & 2.731406 & 1.846757 & 3.869685 \\
\hline 10 & 8 & 0 & 2.240396 & -0.828722 & 4.118380 \\
\hline 11 & 6 & 0 & 1.223170 & 1.574343 & 5.674569 \\
\hline 12 & 9 & 0 & 1.927490 & 0.647470 & 6.349861 \\
\hline 13 & 9 & $\odot$ & ๑. 599979 & 2.260120 & 3.515519 \\
\hline 14 & 9 & $\odot$ & -0.100601 & 1. 346199 & 5.888450 \\
\hline 15 & 6 & $\odot$ & 1.595381 & 2.974464 & 6.277406 \\
\hline 16 & 9 & $\odot$ & 1.205851 & 3.955340 & 5.439415 \\
\hline 17 & 9 & $\odot$ & 2.938124 & 3.026297 & 6.413992 \\
\hline 18 & 6 & $\odot$ & ๑. 939152 & 3.252631 & 7.675848 \\
\hline 19 & 9 & $\odot$ & 0.987474 & 2.131211 & 8.423180 \\
\hline 20 & 9 & $\odot$ & -0.352461 & 3.592279 & 7.477070 \\
\hline 21 & 6 & $\odot$ & 1.640900 & 4.400994 & 8.482345 \\
\hline 22 & 9 & $\odot$ & 1.944499 & 5.411028 & 7.641864 \\
\hline 23 & 9 & $\odot$ & 2.780468 & 3.912001 & 9.013344 \\
\hline 24 & 6 & $\odot$ & $\odot .757906$ & 4.984267 & 9.639823 \\
\hline 25 & 9 & $\odot$ & ๑. 126519 & 3.971918 & 10.270378 \\
\hline 26 & 9 & $\odot$ & -0.166128 & 5.805152 & 9.098031 \\
\hline
\end{tabular}




\begin{tabular}{|c|c|c|c|c|c|}
\hline 27 & 6 & $\odot$ & 1.570338 & 5.785263 & 10.710688 \\
\hline 28 & 9 & $\odot$ & 2.472644 & 6.577391 & 10.093134 \\
\hline 29 & 9 & $\odot$ & 2.220423 & 4.911909 & 11.503931 \\
\hline 30 & 6 & $\odot$ & 0.706229 & 6.701780 & 11.634322 \\
\hline 31 & 9 & $\odot$ & -0.311929 & 6.016763 & 12.165588 \\
\hline 32 & 9 & $\odot$ & ๑. 224031 & 7.745898 & 10.957900 \\
\hline 33 & 9 & $\odot$ & 1.479885 & 7.157702 & 12.624478 \\
\hline 34 & 1 & $\odot$ & 0.236463 & $-\odot .293179$ & 3.946820 \\
\hline 35 & 1 & $\odot$ & 2.181915 & -1.685966 & 3.680031 \\
\hline 36 & 1 & $\odot$ & 3.359593 & ๑ . 051977 & 1.877544 \\
\hline 37 & 1 & $\odot$ & 3.327764 & 0.014103 & -0.601641 \\
\hline 38 & 1 & $\odot$ & 1.162328 & $-\odot .065228$ & -1.818029 \\
\hline 39 & 1 & $\odot$ & -0.970521 & -0.108916 & -0.542748 \\
\hline 40 & 1 & $\odot$ & -0.935300 & -0.068597 & 1.932668 \\
\hline
\end{tabular}

(2) $-P 4$

\begin{tabular}{|c|c|c|c|c|c|}
\hline \multirow{2}{*}{$\begin{array}{l}\text { Center } \\
\text { Number }\end{array}$} & \multirow{2}{*}{$\begin{array}{l}\text { Atomic } \\
\text { Number }\end{array}$} & \multirow{2}{*}{$\begin{array}{c}\text { Atomic } \\
\text { Type }\end{array}$} & \multicolumn{3}{|c|}{ Coordinates (Angstroms) } \\
\hline & & & $\mathrm{X}$ & Y & Z \\
\hline \multicolumn{6}{|c|}{ 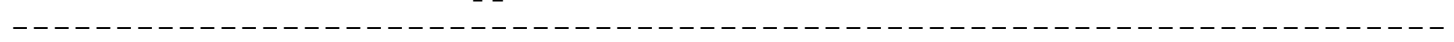 } \\
\hline 1 & 6 & $\odot$ & -.073636 & .087381 & .008725 \\
\hline 2 & 6 & $\odot$ & .045163 & .106292 & 1.401004 \\
\hline 3 & 6 & $\odot$ & 1.316759 & .101766 & 1.983582 \\
\hline 4 & 6 & $\odot$ & 2.456989 & .055939 & 1.184101 \\
\hline 5 & 6 & $\odot$ & 2.335620 & .028065 & -.205457 \\
\hline 6 & 6 & $\odot$ & 1.069166 & .048727 & - . 789194 \\
\hline 7 & 6 & $\odot$ & -1.184410 & .157347 & 2.281608 \\
\hline 8 & 8 & $\odot$ & -2.267887 & .681136 & 1.558869 \\
\hline 9 & 6 & $\odot$ & -1.561893 & -1.196206 & 2.942110 \\
\hline 10 & 6 & 0 & -1.727583 & -2.448505 & 2.027995 \\
\hline 11 & 9 & $\odot$ & -2.410999 & -2.097718 & .917479 \\
\hline 12 & 9 & $\odot$ & -2.753726 & -1.001960 & 3.597120 \\
\hline 13 & 9 & $\odot$ & -.628869 & -1.489904 & 3.883906 \\
\hline 14 & 6 & $\odot$ & -2.489075 & -3.648447 & 2.693731 \\
\hline 15 & 9 & $\odot$ & -2.072368 & -3.788604 & 3.968867 \\
\hline 16 & 9 & $\odot$ & -.495938 & -2.872133 & 1.666092 \\
\hline 17 & 6 & $\odot$ & -2.273163 & -5.018604 & 1.961209 \\
\hline 18 & 9 & $\odot$ & -2.331321 & -4.820591 & .628356 \\
\hline 19 & 9 & $\odot$ & -3.810494 & -3.366941 & 2.692703 \\
\hline 20 & 6 & $\odot$ & -3.340309 & -6.106045 & 2.336692 \\
\hline 21 & 9 & $\odot$ & -3.571952 & -6.056329 & 3.664577 \\
\hline 22 & 9 & $\odot$ & -1.049452 & -5.486239 & 2.284736 \\
\hline 23 & 6 & $\odot$ & -2.900384 & -7.567449 & 1.976339 \\
\hline 24 & 9 & $\odot$ & -2.311325 & -7.566639 & .762365 \\
\hline 25 & 9 & $\odot$ & -4.484352 & -5.824869 & 1.679327 \\
\hline 26 & 6 & $\odot$ & -4.083696 & -8.590916 & 1.936204 \\
\hline 27 & 9 & $\odot$ & -4.899156 & -8.379816 & 2.991417 \\
\hline 28 & 9 & $\odot$ & -2.004223 & -7.980873 & 2.896540 \\
\hline 29 & 6 & $\odot$ & -3.650590 & -10.090985 & 1.977814 \\
\hline 30 & 9 & $\odot$ & -2.726972 & -10.343415 & 1.044635 \\
\hline 31 & 9 & $\odot$ & -4.779215 & -8.404005 & .797867 \\
\hline 32 & 9 & $\odot$ & -4.723653 & -10.850642 & 1.736515 \\
\hline 33 & 9 & $\odot$ & -3.161534 & -10.416253 & 3.175844 \\
\hline
\end{tabular}




$\begin{array}{rrrrrr}34 & 1 & 0 & -.957078 & .782021 & 3.161077 \\ 35 & 1 & 0 & -3.033824 & .740023 & 2.142946 \\ 36 & 1 & 0 & 1.418626 & .134456 & 3.065178 \\ 37 & 1 & 0 & 3.439662 & .051144 & 1.647256 \\ 38 & 1 & 0 & 3.224294 & -.001892 & -.829755 \\ 39 & 1 & 0 & .967678 & .036747 & -1.870845 \\ 40 & 1 & 0 & -1.059360 & .114387 & -.442775\end{array}$

(2)-P1

\begin{tabular}{|c|c|c|c|c|c|}
\hline \multirow{2}{*}{$\begin{array}{l}\text { Center } \\
\text { Number }\end{array}$} & \multirow{2}{*}{$\begin{array}{l}\text { Atomic } \\
\text { Number }\end{array}$} & \multirow{2}{*}{$\begin{array}{c}\text { Atomic } \\
\text { Type }\end{array}$} & \multicolumn{3}{|c|}{ Coordinates (Angstroms) } \\
\hline & & & $\mathrm{x}$ & $\mathrm{Y}$ & Z \\
\hline 1 & 6 & $\odot$ & - . .051842 & .633274 & - . .028445 \\
\hline 2 & 6 & $\odot$ & -.011478 & .455616 & 1.352751 \\
\hline 3 & 6 & 0 & 1.204598 & .188098 & 1.990400 \\
\hline 4 & 6 & $\odot$ & 2.376175 & .120870 & 1.232246 \\
\hline 5 & 6 & $\odot$ & 2.334976 & .295572 & -.150323 \\
\hline 6 & 6 & 0 & 1.119064 & .549596 & -.783586 \\
\hline 7 & 6 & 0 & 1.266380 & .024174 & 3.490744 \\
\hline 8 & 6 & $\odot$ & .671198 & -1.302366 & 4.039533 \\
\hline 9 & 9 & 0 & .633661 & -1.176617 & 5.406667 \\
\hline 10 & 8 & $\odot$ & .552946 & 1.088759 & 4.082607 \\
\hline 11 & 6 & $\odot$ & 1.492135 & -2.588122 & 3.728360 \\
\hline 12 & 9 & $\odot$ & 2.705047 & -2.450349 & 4.325709 \\
\hline 13 & 9 & $\odot$ & -.602884 & -1.493467 & 3.621030 \\
\hline 14 & 9 & $\odot$ & 1.680995 & -2.676962 & 2.395286 \\
\hline 15 & 6 & 0 & .846643 & -3.938216 & 4.200051 \\
\hline 16 & 9 & 0 & -.137506 & -4.254713 & 3.331558 \\
\hline 17 & 9 & $\odot$ & .311751 & -3.785841 & 5.427216 \\
\hline 18 & 6 & $\odot$ & 1.863609 & -5.132011 & 4.264899 \\
\hline 19 & 9 & $\odot$ & 2.601425 & -4.997613 & 5.387318 \\
\hline 20 & 9 & $\odot$ & 2.680949 & -5.078219 & 3.193404 \\
\hline 21 & 6 & $\odot$ & 1.176688 & -6.542320 & 4.280636 \\
\hline 22 & 9 & $\odot$ & .775774 & -6.832215 & 3.025921 \\
\hline 23 & 9 & $\odot$ & .098454 & -6.498886 & 5.089425 \\
\hline 24 & 6 & $\odot$ & 2.116720 & -7.691513 & 4.786001 \\
\hline 25 & 9 & 0 & 2.197872 & -7.614768 & 6.130728 \\
\hline 26 & 9 & $\odot$ & 3.348587 & -7.517379 & 4.263161 \\
\hline 27 & 6 & $\odot$ & 1.626075 & -9.125101 & 4.395766 \\
\hline 28 & 9 & $\odot$ & 1.891481 & -9.333333 & 3.091698 \\
\hline 29 & 9 & $\odot$ & .293785 & -9.215045 & 4.595563 \\
\hline 30 & 6 & $\odot$ & 2.294192 & -10.285380 & 5.200510 \\
\hline 31 & 9 & 0 & 1.872935 & -10.294429 & 6.466475 \\
\hline 32 & 9 & $\odot$ & 3.626270 & -10.169863 & 5.183369 \\
\hline 33 & 9 & $\odot$ & 1.956599 & -11.446645 & 4.631459 \\
\hline 34 & 1 & $\odot$ & 2.318899 & .028982 & 3.808612 \\
\hline 35 & 1 & $\odot$ & .598651 & 1.000712 & 5.043663 \\
\hline 36 & 1 & $\odot$ & 3.327733 & - . . $07 \odot 422$ & 1.722901 \\
\hline 37 & 1 & $\odot$ & 3.252465 & .239786 & -.729235 \\
\hline 38 & 1 & $\odot$ & 1.084255 & .689226 & -1.860496 \\
\hline 39 & 1 & $\odot$ & $-1.00 \odot 216$ & .841062 & -.516009 \\
\hline $4 \odot$ & 1 & $\odot$ & -.919219 & .537781 & 1.940934 \\
\hline
\end{tabular}


(2)-M2

\begin{tabular}{|c|c|c|c|c|c|}
\hline \multirow{2}{*}{$\begin{array}{l}\text { Center } \\
\text { Number }\end{array}$} & \multirow{2}{*}{$\begin{array}{l}\text { Atomic } \\
\text { Number }\end{array}$} & \multirow{2}{*}{$\begin{array}{l}\text { Atomic } \\
\text { Type }\end{array}$} & \multicolumn{3}{|c|}{ Coordinates (Angstroms) } \\
\hline & & & $\mathrm{x}$ & Y & Z \\
\hline 1 & 6 & $\odot$ & 0.000000 & 0.000000 & $\odot .0 \odot \odot \odot \odot \odot$ \\
\hline 2 & 6 & $\odot$ & 1.338672 & $\odot .332029$ & $\odot .195219$ \\
\hline 3 & 6 & 0 & 2.229989 & -0.614080 & 0.718843 \\
\hline 4 & 6 & 0 & 1.762661 & -1.894935 & 1.029256 \\
\hline 5 & 6 & $\odot$ & $\odot .421354$ & -2.225019 & $\odot .836701$ \\
\hline 6 & 6 & $\odot$ & -0.462515 & -1.277087 & 0.323000 \\
\hline 7 & 6 & 0 & 3.697990 & -0.282491 & 0.892415 \\
\hline 8 & 6 & $\odot$ & $4.0 \odot 7259$ & $\odot .712306$ & 2.033179 \\
\hline 9 & 9 & 0 & 5.353640 & 0.910632 & 2.077261 \\
\hline 10 & 8 & $\odot$ & 4.257548 & 0.326235 & -0.256079 \\
\hline 11 & 6 & $\odot$ & 3.564089 & $\odot .230320$ & 3.450486 \\
\hline 12 & 9 & $\odot$ & 3.884619 & -1.082833 & 3.576295 \\
\hline 13 & 9 & $\odot$ & 3.421299 & 1.912126 & 1.800035 \\
\hline 14 & 9 & $\odot$ & 2.222787 & $\odot .355926$ & 3.555424 \\
\hline 15 & 6 & $\odot$ & 4.231234 & $\odot .996085$ & 4.645164 \\
\hline 16 & 9 & 0 & 4.264846 & 2.313814 & 4.364424 \\
\hline 17 & 9 & 0 & 5.494699 & $\odot .543682$ & 4.792099 \\
\hline 18 & 6 & 0 & 3.477754 & $\odot .811051$ & 6.008998 \\
\hline 19 & 9 & $\odot$ & 3.108703 & -0.480784 & 6.136965 \\
\hline 20 & 9 & $\odot$ & 2.372834 & 1.585389 & 5.994754 \\
\hline 21 & 6 & 0 & 4.341438 & 1.194133 & 7.261621 \\
\hline 22 & 9 & 0 & 5.023061 & 2.327210 & 6.997045 \\
\hline 23 & 9 & 0 & 5.218495 & $\odot .196045$ & 7.495806 \\
\hline 24 & 6 & $\odot$ & 3.493273 & 1.430168 & 8.559348 \\
\hline 25 & 9 & $\odot$ & 2.538410 & $\odot .480178$ & 8.643273 \\
\hline 26 & 9 & $\odot$ & 2.900079 & 2.638580 & 8.466809 \\
\hline 27 & 6 & 0 & 4.332750 & 1.385591 & 9.879265 \\
\hline 28 & 9 & 0 & 5.485107 & 2.066686 & 9.704711 \\
\hline 29 & 9 & 0 & 4.619708 & $\odot .101051$ & 10.166750 \\
\hline 30 & 6 & $\odot$ & 3.618364 & 1.994526 & 11.127645 \\
\hline 31 & 9 & 0 & 2.398978 & 1.467298 & 11.279609 \\
\hline 32 & 9 & $\odot$ & 3.510995 & 3.320080 & 11.020592 \\
\hline 33 & 9 & $\odot$ & 4.345829 & 1.713567 & 12.213321 \\
\hline 34 & 1 & 0 & 4.244108 & -1.201305 & 1.146071 \\
\hline 35 & 1 & 0 & 3.885687 & -0.111894 & -1.030703 \\
\hline 36 & 1 & $\odot$ & 2.449885 & -2.638185 & 1.426541 \\
\hline 37 & 1 & 0 & $\odot .070089$ & -3.223011 & 1.083023 \\
\hline 38 & 1 & $\odot$ & -1.507293 & -1.532710 & 0.170118 \\
\hline 39 & 1 & $\odot$ & -0.684536 & $\odot .74112 \odot$ & -0.403034 \\
\hline $4 \odot$ & 1 & 0 & 1.696706 & 1.325503 & -0.057055 \\
\hline
\end{tabular}

(2)-M4

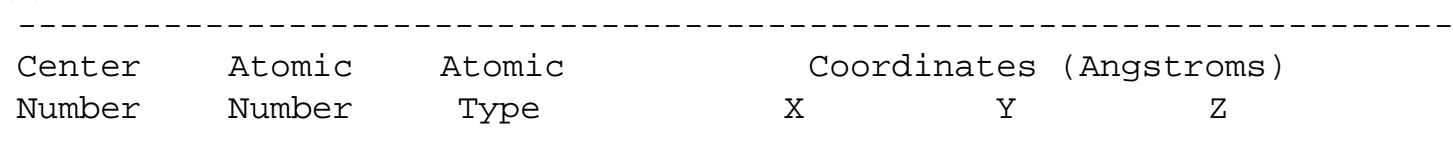




\begin{tabular}{|c|c|c|c|c|c|}
\hline 1 & 6 & $\odot$ & .163847 & 191197 & - .042๑95 \\
\hline 2 & 6 & $\odot$ & .102780 & 184116 & 1.354117 \\
\hline 3 & 6 & $\odot$ & 1.280971 & .330490 & $2.09367 \odot$ \\
\hline 4 & 6 & $\odot$ & 2.508677 & .455505 & 1.445841 \\
\hline 5 & 6 & $\odot$ & 2.567528 & .451305 & .052295 \\
\hline 6 & 6 & $\odot$ & 1.392121 & .324054 & -.687773 \\
\hline 7 & 6 & $\odot$ & -1.226596 & .071109 & 2.069872 \\
\hline 8 & 8 & $\odot$ & -2.272145 & .374008 & 1.180803 \\
\hline 9 & 6 & $\odot$ & -1.504564 & -1.280494 & 2.778208 \\
\hline 10 & 6 & $\odot$ & -1.648496 & -2.565256 & 1.905086 \\
\hline 11 & 9 & $\odot$ & -2.817129 & -2.489976 & 1.225444 \\
\hline 12 & 9 & $\odot$ & -2.691281 & -1.125459 & 3.449218 \\
\hline 13 & 9 & $\odot$ & $-.54 \odot 478$ & -1.503773 & 3.710310 \\
\hline 14 & 6 & $\odot$ & -1.633688 & -3.910290 & 2.715208 \\
\hline 15 & 9 & $\odot$ & -.356141 & -4.188562 & 3.051892 \\
\hline 16 & 9 & $\odot$ & -.633823 & -2.615134 & 1. 018542 \\
\hline 17 & 6 & $\odot$ & -2.218050 & -5.130182 & 1.920176 \\
\hline 18 & 9 & $\odot$ & -3.564585 & -5.036513 & 1.928506 \\
\hline 19 & 9 & $\odot$ & -2.360872 & -3.764653 & 3.841773 \\
\hline 20 & 6 & $\odot$ & -1.809900 & -6.522643 & 2.516807 \\
\hline 21 & 9 & $\odot$ & -.535811 & -6.781998 & 2.157794 \\
\hline 22 & 9 & $\odot$ & -1.780734 & -5.078599 & .645472 \\
\hline 23 & 6 & $\odot$ & -2.718627 & -7.704434 & 2.029886 \\
\hline 24 & 9 & $\odot$ & -3.886414 & -7.650352 & 2.703907 \\
\hline 25 & 9 & $\odot$ & -1.886018 & -6.465382 & 3.862257 \\
\hline 26 & 6 & $\odot$ & -2.083075 & -9.117994 & 2.245203 \\
\hline 27 & 9 & $\odot$ & -1.138590 & -9.315838 & 1. 305402 \\
\hline 28 & 9 & 0 & -2.967279 & -7.557645 & .711818 \\
\hline 29 & 6 & $\odot$ & -3.091161 & -10.307787 & 2.153614 \\
\hline 30 & 9 & $\odot$ & -3.909638 & -10.324096 & 3.207309 \\
\hline 31 & 9 & 0 & -1.509399 & -9.173241 & 3.466245 \\
\hline 32 & 9 & $\odot$ & -2.398764 & -11.450944 & 2.135313 \\
\hline 33 & 9 & $\odot$ & -3.819476 & -10.229084 & 1.034946 \\
\hline 34 & 1 & 0 & -1.216671 & .771149 & 2.921698 \\
\hline 35 & 1 & $\odot$ & -3.107245 & .373582 & 1.664544 \\
\hline 36 & 1 & $\odot$ & 1.242986 & .349387 & 3.179378 \\
\hline 37 & 1 & $\odot$ & 3.417112 & .565836 & 2.031276 \\
\hline 38 & 1 & 0 & 3.523368 & .554541 & -.453755 \\
\hline 39 & 1 & $\odot$ & 1.428948 & .329307 & -1.773610 \\
\hline 40 & 1 & $\odot$ & -.751497 & .098918 & -.616381 \\
\hline
\end{tabular}

(2)-P2

\begin{tabular}{|c|c|c|c|c|c|}
\hline Center & Atomic & Atomic & \multicolumn{3}{|c|}{ Coordinates (Angstroms) } \\
\hline Number & Number & Type & $x$ & $\mathrm{Y}$ & Z \\
\hline 1 & 6 & 0 & -0.050187 & $\odot .082820$ & 0.011136 \\
\hline 2 & 6 & $\odot$ & -0.020604 & ๑. 050959 & 1.403873 \\
\hline 3 & 6 & $\odot$ & 1.205404 & 0.012664 & 2.081876 \\
\hline 4 & 6 & $\odot$ & 2.393414 & $\odot .024588$ & 1.343882 \\
\hline 5 & 6 & $\odot$ & 2.362669 & $\odot .053709$ & $-\odot .049654$ \\
\hline 6 & 6 & $\odot$ & 1.139605 & ๑.๑8०694 & $-\odot .718756$ \\
\hline 7 & 6 & $\odot$ & 1.263035 & $\odot .043280$ & 3.594477 \\
\hline
\end{tabular}




\begin{tabular}{|c|c|c|c|c|c|}
\hline 8 & 6 & $\odot$ & 0.706201 & -1.206111 & 4.318361 \\
\hline 9 & 9 & $\odot$ & $\odot .703470$ & -0.950835 & 5.651499 \\
\hline 10 & 8 & $\odot$ & $\odot .518566$ & 1.120981 & 4.134776 \\
\hline 11 & 6 & 0 & 1.544746 & -2.504177 & 4.104413 \\
\hline 12 & 9 & 0 & 2.768030 & -2.296353 & 4.656937 \\
\hline 13 & 9 & 0 & -0.574779 & -1.467261 & 3.945466 \\
\hline 14 & 9 & $\odot$ & 1.709509 & -2.718335 & 2.780515 \\
\hline 15 & 6 & $\odot$ & $\odot .929163$ & -3.812349 & 4.713629 \\
\hline 16 & 9 & $\odot$ & $-\odot .063691$ & -4.225461 & 3.895914 \\
\hline 17 & 9 & 0 & 0.412447 & -3.554820 & 5.929930 \\
\hline 18 & 6 & $\odot$ & 1.965791 & -4.980500 & 4.870653 \\
\hline 19 & 9 & 0 & 2.722004 & -4.731962 & 5.960562 \\
\hline 20 & 9 & 0 & 2.762880 & -5.017998 & 3.782828 \\
\hline 21 & 6 & $\odot$ & 1.300808 & -6.392312 & 5.031938 \\
\hline 22 & 9 & 0 & ๑.881805 & -6.804943 & 3.817774 \\
\hline 23 & 9 & 0 & $\odot .236942$ & -6.288531 & 5.853894 \\
\hline 24 & 6 & 0 & 2.266856 & -7.476738 & 5.623657 \\
\hline 25 & 9 & 0 & 2.373650 & -7.272735 & 6.953158 \\
\hline 26 & 9 & 0 & 3.485653 & -7.338232 & 5.060871 \\
\hline 27 & 6 & 0 & 1.788979 & -8.946831 & 5.380597 \\
\hline 28 & 9 & 0 & 2.033368 & -9.275039 & 4.097123 \\
\hline 29 & 9 & $\odot$ & $\odot .461970$ & -9.034161 & 5.613762 \\
\hline 30 & 6 & 0 & 2.487682 & -10.017312 & 6.278407 \\
\hline 31 & 9 & 0 & 2.088178 & -9.912781 & 7.547064 \\
\hline 32 & 9 & $\odot$ & 3.817510 & -9.886975 & 6.226076 \\
\hline 33 & 9 & 0 & 2.157537 & -11.231477 & 5.827235 \\
\hline 34 & 1 & $\odot$ & 2.314769 & 0.115527 & 3.907723 \\
\hline 35 & 1 & $\odot$ & $\odot .517890$ & 1.830901 & 3.481959 \\
\hline 36 & 1 & 0 & 3.349194 & $0.0 \odot 4693$ & 1.862506 \\
\hline 37 & 1 & 0 & 3.293017 & ๑.059408 & -0.610372 \\
\hline 38 & 1 & 0 & 1.112616 & 0.104661 & $-1.8 \odot 4534$ \\
\hline 39 & 1 & 0 & -1.005669 & 0.106959 & -0.505315 \\
\hline 40 & 1 & $\odot$ & $-\odot .948718$ & 0.053370 & 1.966969 \\
\hline
\end{tabular}

(2)-P5

\begin{tabular}{|c|c|c|c|c|c|}
\hline \multirow{2}{*}{$\begin{array}{l}\text { Center } \\
\text { Number }\end{array}$} & \multirow{2}{*}{$\begin{array}{l}\text { Atomic } \\
\text { Number }\end{array}$} & \multirow{2}{*}{$\begin{array}{l}\text { Atomic } \\
\text { Type }\end{array}$} & \multicolumn{3}{|c|}{ Coordinates (Angstroms) } \\
\hline & & & $\mathrm{X}$ & $\mathrm{Y}$ & Z \\
\hline 1 & 6 & 0 & -.104914 & .173484 & .023394 \\
\hline 2 & 6 & $\odot$ & .045429 & .069895 & 1.410194 \\
\hline 3 & 6 & 0 & 1.327982 & -.068599 & 1.951440 \\
\hline 4 & 6 & 0 & 2.443668 & -.121867 & 1.118122 \\
\hline 5 & 6 & 0 & 2.288133 & -.023385 & -.264922 \\
\hline 6 & 6 & 0 & 1.012682 & .128206 & -.808934 \\
\hline 7 & 6 & 0 & -1.160531 & .148392 & 2.329944 \\
\hline 8 & 8 & 0 & -2.232593 & .890117 & 1.798187 \\
\hline 9 & 6 & 0 & -1.729054 & -1.219844 & 2.773413 \\
\hline 10 & 6 & $\Theta$ & -2.170051 & -2.228785 & 1.665730 \\
\hline 11 & 9 & 0 & -2.863578 & -1.574139 & .711111 \\
\hline 12 & 9 & $\odot$ & -2.795180 & -.999687 & 3.586470 \\
\hline 13 & 9 & $\Theta$ & -.766408 & -1.833729 & 3.517546 \\
\hline 14 & 6 & 0 & -3.068279 & -3.407390 & 2.180732 \\
\hline
\end{tabular}




\begin{tabular}{|c|c|c|c|c|c|}
\hline 15 & 9 & 0 & -2.583844 & -3.858236 & 3.356169 \\
\hline 16 & 9 & 0 & -1.055685 & -2.755520 & 1.105318 \\
\hline 17 & 6 & $\odot$ & -3.139198 & -4.623948 & 1.192373 \\
\hline 18 & 9 & 0 & -3.257909 & -4.163890 & - . 070193 \\
\hline 19 & 9 & 0 & -4.319887 & -2.938956 & 2.371148 \\
\hline 20 & 6 & $\odot$ & -4.344922 & -5.588850 & 1.470364 \\
\hline 21 & 9 & 0 & -4.473151 & -5.764054 & 2.801499 \\
\hline 22 & 9 & 0 & -1.992143 & -5.327356 & 1.298027 \\
\hline 23 & 6 & 0 & -4.181898 & -7.002182 & .810706 \\
\hline 24 & 9 & 0 & -3.681914 & -6.856660 & -.434326 \\
\hline 25 & 9 & 0 & -5.468468 & -5.016167 & .991193 \\
\hline 26 & 6 & 0 & -5.520648 & -7.805001 & .699628 \\
\hline 27 & 9 & 0 & -6.219651 & -7.679288 & 1.847813 \\
\hline 28 & 9 & $\odot$ & -3.310086 & -7.719175 & 1.550262 \\
\hline 29 & 6 & 0 & -5.344241 & -9.333291 & .429965 \\
\hline 30 & 9 & $\odot$ & -4.534193 & -9.540544 & -.613518 \\
\hline 31 & 9 & $\odot$ & -6.246464 & - 7.298159 & -.315819 \\
\hline 32 & 9 & $\odot$ & -6.542327 & -9.858149 & .154004 \\
\hline 33 & 9 & 0 & -4.845567 & -9.954948 & 1.500089 \\
\hline 34 & 1 & $\odot$ & -.833770 & .571143 & 3.293785 \\
\hline 35 & 1 & 0 & -1.916923 & 1.782756 & 1.614266 \\
\hline 36 & 1 & 0 & 1.454836 & -.137609 & 3.028919 \\
\hline 37 & 1 & $\odot$ & 3.434470 & - . 232095 & 1.549791 \\
\hline 38 & 1 & 0 & 3.157796 & - . .059288 & -.915041 \\
\hline 39 & 1 & $\odot$ & .885106 & .208060 & -1.884904 \\
\hline 40 & 1 & $\odot$ & -1.098446 & .285204 & -.400186 \\
\hline
\end{tabular}

(2)-M5

\begin{tabular}{|c|c|c|c|c|c|}
\hline \multirow{2}{*}{$\begin{array}{l}\text { Center } \\
\text { Number }\end{array}$} & \multirow{2}{*}{$\begin{array}{l}\text { Atomic } \\
\text { Number }\end{array}$} & \multirow{2}{*}{$\begin{array}{c}\text { Atomic } \\
\text { Type }\end{array}$} & \multicolumn{3}{|c|}{ Coordinates (Angstroms) } \\
\hline & & & $\mathrm{X}$ & Y & Z \\
\hline \multicolumn{6}{|c|}{ - - - - - - - - - - - - - - - - - - - - - - - - - - - - - - - - - - - - - - - - - - - } \\
\hline 1 & 6 & $\odot$ & 0.000780 & -0.004063 & -0.002050 \\
\hline 2 & 6 & $\odot$ & -0.000502 & -0.000921 & 1.392822 \\
\hline 3 & 6 & $\odot$ & 1.203007 & ๑. . 00693 & 2.096592 \\
\hline 4 & 6 & $\odot$ & 2.421562 & ๑ . 001804 & 1.410339 \\
\hline 5 & 6 & $\odot$ & 2.416288 & ๑. 015859 & 0. 011089 \\
\hline 6 & 6 & $\odot$ & 1. 213176 & ๑.๑๑3884 & -0.692178 \\
\hline 7 & 6 & $\odot$ & 3.742784 & 0.060727 & 2.158204 \\
\hline 8 & 8 & $\odot$ & 3.640021 & $\odot .606187$ & 3.452969 \\
\hline 9 & 6 & $\odot$ & 4.500643 & -1.279083 & 2.312834 \\
\hline 10 & 9 & $\odot$ & 5.782588 & -0.995369 & 2.666018 \\
\hline 11 & 9 & $\odot$ & 4.540603 & -1.886048 & 1.093357 \\
\hline 12 & 6 & $\odot$ & 3.959184 & -2.313169 & 3.351262 \\
\hline 13 & 6 & $\odot$ & 4.523782 & -3.766780 & 3.170939 \\
\hline 14 & 6 & $\odot$ & 4.325983 & -4.678204 & 4.432867 \\
\hline 15 & 6 & $\odot$ & 4.472944 & -6.210714 & 4.131129 \\
\hline 16 & 6 & $\odot$ & 4.708536 & -7.082759 & 5.413398 \\
\hline 17 & 6 & $\odot$ & 4.417869 & -8.605738 & 5.201576 \\
\hline 18 & 6 & $\odot$ & 5.008246 & -9.543460 & 6.302290 \\
\hline 19 & 9 & $\odot$ & 4.524268 & -10.775405 & 6.114259 \\
\hline 20 & 9 & $\odot$ & 2.613770 & -2.385297 & 3.240890 \\
\hline 21 & 9 & $\odot$ & 4.272096 & -1.889597 & 4.593290 \\
\hline
\end{tabular}




\begin{tabular}{|c|c|c|c|c|c|}
\hline 22 & 9 & 0 & 5.844977 & -3.704527 & 2.908053 \\
\hline 23 & 9 & 0 & 3.894613 & -4.344907 & 2.124822 \\
\hline 24 & 9 & 0 & 3.092674 & $-4.4712 \odot 4$ & 4.938927 \\
\hline 25 & 9 & $\odot$ & 5.245886 & -4.333493 & 5.357923 \\
\hline 26 & 9 & 0 & 5.519614 & -6.399187 & 3.301705 \\
\hline 27 & 9 & 0 & 3.346445 & -6.636138 & 3.522489 \\
\hline 28 & 9 & $\odot$ & 3.904933 & -6.642365 & 6.403957 \\
\hline 29 & 9 & 0 & 5.994005 & -6.938631 & 5.797431 \\
\hline 30 & 9 & $\odot$ & 4.933109 & -9.003985 & 4.018742 \\
\hline 31 & 9 & $\odot$ & 3.083912 & -8.793751 & 5.186707 \\
\hline 32 & 9 & 0 & 4.654991 & -9.125182 & 7.522147 \\
\hline 33 & 9 & $\odot$ & 6.339454 & -9.594696 & 6.226770 \\
\hline 34 & 1 & 0 & 4.451705 & $\odot .640008$ & 1.544727 \\
\hline 35 & 1 & 0 & 3.302576 & 1.506433 & 3.376917 \\
\hline 36 & 1 & $\odot$ & 3.357248 & $\odot .035072$ & -0.532995 \\
\hline 37 & 1 & $\odot$ & 1.223469 & ๑. 010710 & -1.778442 \\
\hline 38 & 1 & 0 & -0.938071 & -0.006469 & -0.548681 \\
\hline 39 & 1 & 0 & $-\odot .940879$ & -0.003436 & 1.936946 \\
\hline 40 & 1 & 0 & 1.199930 & $-\odot .00634 \odot$ & 3.182186 \\
\hline
\end{tabular}

